DOI 10.4171/JEMS/378

M. Boulakia $\cdot$ S. Guerrero

\title{
Local null controllability of a fluid-solid interaction problem in dimension 3
}

Received December 17, 2010 and in revised form July 20, 2011

\begin{abstract}
We are interested in the three-dimensional coupling between an incompressible fluid and a rigid body. The fluid is modeled by the Navier-Stokes equations, while the solid satisfies Newton's laws. In the main result of the paper we prove that, with the help of a distributed control, we can drive the fluid and structure velocities to zero and the solid to a reference position provided that the initial velocities are small enough and the initial position of the structure is close to the reference position. This is done without any condition on the geometry of the rigid body.
\end{abstract}

\section{Introduction}

\subsection{Statement of problem}

We consider a rigid structure immersed in a viscous incompressible fluid. At time $t$, we denote by $\Omega_{S}(t)$ the domain occupied by the structure. The structure and the fluid are contained in a fixed bounded domain $\Omega \subset \mathbb{R}^{3}$. Let $\mathcal{O} \subset \subset \Omega$ be the control domain. We suppose that the boundaries of $\Omega_{S}(0)$ and $\Omega$ are smooth $\left(C^{4}\right.$ for instance) and that

$$
\Omega_{S}(0) \subset \Omega \backslash \mathcal{O}, \quad d\left(\partial(\Omega \backslash \mathcal{O}), \overline{\Omega_{S}(0)}\right) \geq \delta_{0}>0 .
$$

For any $t>0$, we denote by $\Omega_{F}(t):=\Omega \backslash \overline{\Omega_{S}(t)}$ the region occupied by the fluid and by $\widetilde{\mathcal{O}} \subset \subset \mathcal{O}$ an open set. The time evolution of the eulerian velocity $u$ and the pressure $p$ of the fluid is governed by the incompressible Navier-Stokes equations: for all $t>0$ and $x \in \Omega_{F}(t)$,

$$
\left\{\begin{array}{l}
\left(u_{t}+(u \cdot \nabla) u\right)(t, x)-\nabla \cdot \sigma(u, p)(t, x)=v(t, x) \zeta(x), \\
\nabla \cdot u(t, x)=0 .
\end{array}\right.
$$

The stress tensor is given by

$$
\sigma(u, p):=2 \mu \epsilon(u)-p \mathrm{Id},
$$

where $\epsilon(u):=\frac{1}{2}\left(\nabla u+\nabla u^{t}\right)$ and the viscosity coefficient $\mu$ is supposed to be positive. The function $\zeta \in C_{c}^{2}(\mathcal{O})$ satisfies $\zeta=1$ in $\widetilde{\mathcal{O}}$ and $v$ is a control force which acts over the system through $\mathcal{O}$.

M. Boulakia, S. Guerrero: Université Pierre et Marie Curie-Paris 6, UMR 7598 Laboratoire Jacques-Louis Lions, F-75005 Paris, France; e-mails: boulakia@ann.jussieu.fr, guerrero@ann.jussieu.fr 
At time $t$, the motion of the rigid structure is given by the position $b(t) \in \mathbb{R}^{3}$ of the center of mass and by a rotation (orthogonal) matrix $Q(t) \in \mathbb{M}_{3 \times 3}(\mathbb{R})$. The domain $\Omega_{S}(t)$ is given by $\chi_{S}\left(t, \Omega_{S}(0)\right)$, where $\chi_{S}$ denotes the flow associated to the motion of the structure:

$$
\chi_{S}(t, y)=b(t)+Q(t) Q_{0}^{-1}\left(y-b_{0}\right) \quad \forall y \in \Omega_{S}(0) .
$$

Here, $Q_{0}$ and $b_{0}$ are the initial rotation matrix and the initial position of the solid.

Let $r:(0, T) \rightarrow \mathbb{R}^{3}$ be the angular velocity. Then the rotation matrix is the solution of the following system:

$$
\left\{\begin{array}{l}
\frac{d Q}{d t}(t)=(r \times Q)(t), \quad t \in(0, T), \\
Q(0)=Q_{0} .
\end{array}\right.
$$

For the equations of the structure, we denote by $m>0$ the mass of the rigid structure and by $J(t) \in \mathbb{M}_{3 \times 3}(\mathbb{R})$ its inertia tensor at time $t$. This tensor is given by

$$
J(t) d \cdot \tilde{d}=\int_{\Omega_{S}(0)}\left(d \times Q(t)\left(y-b_{0}\right)\right) \cdot\left(\tilde{d} \times Q(t)\left(y-b_{0}\right)\right) d y \quad \forall d, \tilde{d} \in \mathbb{R}^{3} .
$$

One can prove that

$$
J(t) d \cdot d \geq C_{J}|d|^{2} \quad \text { for all } d \in \mathbb{R}^{3},
$$

where $C_{J}$ is a positive constant independent of $t>0$. The equations of the structure motion are given by the balance of linear and angular momentum. We have, for all $t \in$ $(0, T)$,

$$
\left\{\begin{array}{l}
m \ddot{b}=\int_{\partial \Omega_{S}(t)} \sigma(u, p) n d \gamma, \\
J \dot{r}=(J r) \times r+\int_{\partial \Omega_{S}(t)}(x-b) \times(\sigma(u, p) n) d \gamma .
\end{array}\right.
$$

In these equations, $n$ is the outward unit normal to $\partial \Omega_{S}(t)$. On the boundary of the fluid, the eulerian velocity has to satisfy a no-slip boundary condition. Therefore, we have, for all $t>0$,

$$
\left\{\begin{array}{l}
u(t, x)=0, \quad \forall x \in \partial \Omega, \\
u(t, x)=\dot{b}(t)+r(t) \times(x-b(t)), \quad \forall x \in \partial \Omega_{S}(t) .
\end{array}\right.
$$

The system is completed with the following initial conditions:

$$
u(0, \cdot)=u_{0} \text { in } \Omega_{F}(0), \quad b(0)=b_{0}, \dot{b}(0)=b_{1}, r(0)=r_{0},
$$

which satisfy $u_{0} \in H^{1}\left(\Omega_{F}(0)\right)$ and

$\nabla \cdot u_{0}=0$ in $\Omega_{F}(0), \quad u_{0}=0$ on $\partial \Omega, \quad u_{0}(x)=b_{1}+r_{0} \times\left(x-b_{0}\right), x \in \partial \Omega_{S}(0)$.

Let us now recall some of the most relevant results on interaction problems between a rigid structure and an incompressible fluid. 
A local result was proved in [12], while the existence of global weak solutions was proved in [5] and [6] (with variable density) and [16] (2D, with variable density); in this last paper, the existence of a solution was proved even beyond collisions. Later, the existence and uniqueness of strong global solutions in 2D was proved in [17] together with the local in time existence and uniqueness of strong solutions in $3 D$.

In this paper, we prove the local null controllability of system (2)-(7). The same result was proved in [4] and in [14] in dimension 2 provided that $\Omega_{S}(0)$ satisfies some geometric conditions. For the Burgers equation with a moving particle in dimension 1, the local null controllability was proved in [7]. In the absence of a solid, the local exact controllability to the trajectories of the Navier-Stokes equations was proved in [13]. This result was later improved in [9].

We now state the main result of this paper:

Theorem 1. There exists $\delta>0$ such that for any $\left(u_{0}, b_{0}, b_{1}, r_{0}, Q_{0}\right)$ satisfying (8), $u_{0} \in$ $H^{2}\left(\Omega_{F}(0)\right)$ and

$$
\left\|u_{0}\right\|_{H^{2}\left(\Omega_{F}(0)\right)}+\left|b_{0}\right|+\left|b_{1}\right|+\left|r_{0}\right|+\left|Q_{0}-\mathrm{Id}\right|<\delta
$$

there exists a control $v \in L^{2}\left(0, T ; H^{1}(\Omega)\right)$ such that the solution of (2)-(7) satisfies

$$
u(T, \cdot)=0 \text { in } \Omega_{F}(T), \quad b(T)=0, \dot{b}(T)=0, r(T)=0, Q(T)=\mathrm{Id} .
$$

The proof of this result is based on a fixed-point argument. For this, we first consider a linearized system for which we prove the existence of controls in $L^{2}\left(0, T ; H^{1}(\Omega)\right)$ which drive the velocities to zero and the position of the structure to the desired reference position $(b(T), Q(T))=(0, \mathrm{Id})$.

This null controllability result is established with the help of a Carleman inequality for the associated adjoint system. To prove this inequality, we use a method different and more concise than the one presented in [13] and [9] and used in [4] and [14]: we first consider the parabolic equation satisfied by the curl of the solution (where the pressure does not appear) and establish a Carleman inequality for this parabolic problem in terms of two boundary integrals concerning some traces of the velocity. These boundary terms are then estimated thanks to regularity results which are stated and proved in the Appendix at the end of the paper.

\subsection{A problem linearized with respect to the fluid velocity}

Let us introduce

$$
\left\{\begin{array}{l}
(\hat{b}, \hat{r}) \in H^{2}(0, T) \times H^{1}(0, T), \\
(\hat{b}, \hat{\hat{b}}, \hat{r})_{\mid t=0}=\left(b_{0}, b_{1}, r_{0}\right) .
\end{array}\right.
$$

This allows us to define the domains

$$
\widehat{\Omega}_{S}(t):=\hat{b}(t)+\widehat{Q}(t) Q_{0}^{-1}\left(\Omega_{S}(0)-b_{0}\right) \quad \text { and } \quad \widehat{\Omega}_{F}(t):=\Omega \backslash \overline{\widehat{\Omega}_{S}(t)},
$$


where $\widehat{Q}$ is the solution of (3) with $r$ replaced by $\hat{r}$. We suppose that the solid domain stays far away from $\partial(\Omega \backslash \mathcal{O})$ :

$$
\exists \delta_{1}>0 \forall t \in[0, T]: \quad d\left(\overline{\widehat{\Omega}_{S}(t)}, \partial(\Omega \backslash \mathcal{O})\right) \geq \delta_{1} .
$$

Let us now define several notations which we will use all along the paper. We introduce the following spaces of functions defined on moving domains: for $r, p \in \mathbb{N}$,

$$
\begin{aligned}
L^{2}\left(L^{2}\right) & :=\left\{u \text { measurable }: \int_{0}^{T} \int_{\widehat{\Omega}_{F}(s)}|u|^{2} d x d s<\infty\right\}, \\
L^{2}\left(H^{p}\right) & :=\left\{u \in L^{2}\left(L^{2}\right): \int_{0}^{T}\|u\|_{H^{p}\left(\widehat{\Omega}_{F}(s)\right)}^{2} d s<\infty\right\}, \\
H^{r}\left(H^{p}\right) & :=\left\{u \in L^{2}\left(L^{2}\right): \int_{0}^{T} \sum_{\beta=0}^{r}\left\|\partial_{t}^{\beta} u\right\|_{H^{p}\left(\widehat{\Omega}_{F}(s)\right)}^{2} d s<\infty\right\},
\end{aligned}
$$

with the natural associated norms coming from the definition. On the other hand, we define

$$
\begin{aligned}
C^{0}\left(L^{2}\right) & :=\left\{u: \widetilde{u}(s, x):=u(s, x) 1 \widehat{\Omega}_{F}(s) \in C^{0}\left([0, T] ; L^{2}(\Omega)\right)\right\}, \\
C^{r}\left(H^{p}\right) & :=\left\{u: \partial_{t}^{\beta} \partial_{x}^{\alpha} u \in C^{0}\left(L^{2}\right), \quad \forall 0 \leq \beta \leq r, \forall 0 \leq|\alpha| \leq p\right\},
\end{aligned}
$$

with the associated norms

$$
\begin{aligned}
\|u\|_{C^{0}\left(L^{2}\right)} & :=\max _{t \in(0, T)}\|u(t)\|_{L^{2}\left(\widehat{\Omega}_{F}(t)\right)}=\max _{t \in(0, T)}\|\widetilde{u}(t)\|_{L^{2}(\Omega)}, \\
\|u\|_{C^{r}\left(H^{p}\right)} & :=\sum_{\beta=0}^{r} \max _{t \in(0, T)}\left\|\partial_{t}^{\beta} u(t)\right\|_{H^{p}\left(\widehat{\Omega}_{F}(t)\right)} .
\end{aligned}
$$

Let us now consider a velocity $\hat{u}$ satisfying

$$
\left\{\begin{array}{l}
\hat{u} \in \hat{Z}:=H^{1}\left(L^{6}\right) \cap L^{2}\left(W^{2,6}\right), \quad \nabla \cdot \hat{u}=0, \quad x \in \widehat{\Omega}_{F}(t), \\
\hat{u}(t, x)=(\dot{\hat{b}}(t)+\hat{r}(t) \times(x-\hat{b}(t))) 1_{\partial \widehat{\Omega}_{S}(t)}(x), \quad x \in \partial \widehat{\Omega}_{F}(t) .
\end{array}\right.
$$

Let us also introduce the spaces

$$
\hat{Y}_{k}:=L^{2}\left(H^{2+k}\right) \cap H^{1+k / 2}\left(L^{2}\right)
$$

for $k \in[-2,2]$. Observe that $\hat{Y}_{k}$ is continuously imbedded in $H^{1}\left(H^{k}\right)$. 
Now, we consider the following linear system around $(\hat{u}, \hat{b}, \hat{r})$ : for all $t \in(0, T)$,

$$
\begin{cases}u_{t}(t, x)+(\hat{u} \cdot \nabla) u(t, x)-\nabla \cdot \sigma(u, p)(t, x)=v(t, x) \zeta(x), & x \in \widehat{\Omega}_{F}(t), \\ \nabla \cdot u(t, x)=0, & x \in \widehat{\Omega}_{F}(t), \\ u(t, x)=0, & x \in \partial \Omega, \\ u(t, x)=\dot{b}(t)+r(t) \times(x-\hat{b}(t)), & x \in \partial \widehat{\Omega}_{S}(t), \\ m \ddot{b}(t)=\int_{\partial \widehat{\Omega}_{S}(t)}(\sigma(u, p) n)(t, x) d \gamma, & \\ (\hat{J} \dot{r})(t)=((\hat{J} \hat{r}) \times r)(t)+\int_{\partial \widehat{\Omega}_{S}(t)}(x-\hat{b}(t)) \times(\sigma(u, p) n)(t, x) d \gamma, \\ u_{\mid t=0}=u_{0} \quad \text { in } \Omega_{F}(0), \quad b(0)=b_{0}, \dot{b}(0)=b_{1}, r(0)=r_{0},\end{cases}
$$

where $\hat{J}$ is defined by (4) with $Q$ replaced by $\widehat{Q}$. The rotation matrix $Q$ is then defined by (3).

As we will see in Section 3, we will be interested in driving the solution of (13) to zero by means of $L^{2}\left(0, T ; H^{1}(\Omega)\right)$ controls. In order to do this, we will first obtain $L^{2}((0, T) \times \Omega)$ controls supported in a smaller open set $\mathcal{O}_{2} \subset \subset \widetilde{\mathcal{O}}$ for the system

$$
\begin{cases}u_{t}^{*}(t, x)+(\hat{u} \cdot \nabla) u^{*}(t, x)-\nabla \cdot \sigma\left(u^{*}, p^{*}\right)(t, x)=v^{*}(t, x) 1_{\mathcal{O}_{2}}(x), & x \in \widehat{\Omega}_{F}(t), \\ \nabla \cdot u^{*}(t, x)=0, & x \in \widehat{\Omega}_{F}(t), \\ u^{*}(t, x)=0, & x \in \partial \Omega, \\ u^{*}(t, x)=\dot{b}^{*}(t)+r^{*}(t) \times(x-\hat{b}(t)), & x \in \partial \widehat{\Omega}_{S}(t), \\ m \ddot{b^{*}}(t)=\int_{\partial \widehat{\Omega}_{S}(t)}\left(\sigma\left(u^{*}, p^{*}\right) n\right)(t, x) d \gamma, & \\ \left(\hat{J} r^{*}\right)(t)=\left((\hat{J} \hat{r}) \times r^{*}\right)(t)+\int_{\partial \widehat{\Omega}_{S}(t)}(x-\hat{b}(t)) \times\left(\sigma\left(u^{*}, p^{*}\right) n\right)(t, x) d \gamma, \\ u_{\mid t=0}^{*}=u_{0} \quad \text { in } \Omega_{F}(0), b^{*}(0)=b_{0}, \dot{b}^{*}(0)=b_{1}, r^{*}(0)=r_{0} .\end{cases}
$$

Notice that the control force is slightly different from the one in (13).

In order to prove the null controllability of this system, we will prove a Carleman inequality for its adjoint system, which is

$$
\begin{cases}-\varphi_{t}(t, x)-(\hat{u} \cdot \nabla) \varphi(t, x)-\nabla \cdot \sigma(\varphi, \pi)(t, x)=0, & x \in \widehat{\Omega}_{F}(t), \\ \nabla \cdot \varphi(t, x)=0, & x \in \widehat{\Omega}_{F}(t), \\ \varphi(t, x)=0, & x \in \partial \Omega, \\ \varphi(t, x)=\dot{a}(t)+\omega(t) \times(x-\hat{b}(t)), & x \in \partial \widehat{\Omega}_{S}(t), \\ m \ddot{a}(t)=-\int_{\partial \widehat{\Omega}_{S}(t)}(\sigma(\varphi, \pi) n)(t, x) d \gamma, & \\ \frac{d}{d t}(\hat{J} \omega)(t)=((\hat{J} \hat{r}) \times \omega)(t)-\int_{\partial \widehat{\Omega}_{S}(t)}(x-\hat{b}(t)) \times(\sigma(\varphi, \pi) n)(t, x) d \gamma, \\ \varphi_{\mid t=T}=\varphi_{T} \quad \text { in } \widehat{\Omega}_{F}(T), \quad a(T)=a_{0}^{T}, \dot{a}(T)=a_{1}^{T}, \omega(T)=\omega_{T} .\end{cases}
$$

We will suppose that $\varphi_{T} \in L^{2}\left(\widehat{\Omega}_{F}(T)\right)$ and $a_{0}^{T}, a_{1}^{T}, \omega_{T} \in \mathbb{R}^{3}$. 
The paper is organized as follows: in Section 2, we state and prove the Carleman inequality satisfied by the adjoint system. In Section 3, we deduce from this inequality an observability inequality and a controllability result for the linearized system. Finally, in Section 4, we prove the null controllability of the non-linear system using a fixed point theorem.

\section{Carleman inequality for the adjoint system}

Let us first introduce the weight functions which we will use in the proof. Let $\beta \in$ $C^{0}\left(W^{2, \infty}\right) \cap C^{1}\left(W^{1, \infty}\right)$ satisfy

$$
\begin{gathered}
\beta=0 \quad \text { on } \partial \widehat{\Omega}_{F}(t), \quad \beta>0 \quad \text { in } \widehat{\Omega}_{F}(t), \quad|\nabla \beta| \geq c_{0}>0 \quad \text { in } \widehat{\Omega}_{F}(t) \backslash \overline{\mathcal{O}}_{0}, \\
\frac{\partial \beta}{\partial n} \leq-c_{1}<0 \quad \text { on } \partial \Omega, \quad \frac{\partial \beta}{\partial n} \geq c_{2}>0 \quad \text { on } \partial \widehat{\Omega}_{S}(t),
\end{gathered}
$$

where $\mathcal{O}_{0} \subset \subset \mathcal{O}_{2}$ is an open set. The existence of a function $\beta$ with the above properties is proved in [4]. Let now $\lambda$ be a positive parameter, $M:=\|\beta\|_{C^{0}\left(L^{\infty}\right)}$ and

$$
\begin{aligned}
\alpha(t, x) & :=\frac{e^{(2 k+2) \lambda M}-e^{\lambda(2 k M+\beta(t, x))}}{t^{k}(T-t)^{k}}, & \xi(t, x) & =\frac{e^{\lambda(2 k M+\beta(t, x))}}{t^{k}(T-t)^{k}}, \\
\alpha^{*}(t) & :=\frac{e^{(2 k+2) \lambda M}-e^{2 k \lambda M}}{t^{k}(T-t)^{k}}, & \xi^{*}(t) & =\frac{e^{2 k \lambda M}}{t^{k}(T-t)^{k}} .
\end{aligned}
$$

Here, $k \geq 24$ is a constant.

We will prove the following Carleman inequality:

Proposition 2. Let $(\hat{u}, \hat{b}, \hat{r})$ be such that (10)-(12) are satisfied. Then there exist constants $C_{1}$ (depending on $\Omega, \mathcal{O}, \delta_{0}$ and $\|\hat{u}\|_{\hat{Z}},\|\hat{b}\|_{W^{1, \infty}(0, T)},\|\hat{r}\|_{L^{\infty}(0, T)}$ ) and $C_{2}>0$ (just depending on $\Omega, \mathcal{O}$ and $\left.\delta_{0}\right)$ such that for all $\varphi_{T} \in L^{2}\left(\widehat{\Omega}_{F}(T)\right)$ and all $a_{0}^{T}, a_{1}^{T}, \omega_{T} \in \mathbb{R}^{3}$ we have

$$
\begin{array}{r}
s^{4} \lambda^{6} \int_{0}^{T} \int_{\widehat{\Omega}_{F}(t)} e^{-2 s \alpha} \xi^{5}|\varphi|^{2} d x d t+s^{4} \lambda^{5} \int_{0}^{T} e^{-2 s \alpha^{*}}\left(\xi^{*}\right)^{4}\left(|\dot{a}|^{2}+|\omega|^{2}\right) d t \\
\leq C_{2} s^{5} \lambda^{6} \int_{0}^{T} \int_{\mathcal{O}_{2}} e^{-2 s \alpha} \xi^{5}|\varphi|^{2} d x d t
\end{array}
$$

for all $\lambda \geq C_{1}$ and all $s \geq C_{1}\left(T^{k}+T^{2 k}\right)$, where $(\varphi, \pi, a, \omega)$ is the solution to (15).

Proof. All along the proof, $C$ (resp. $\widehat{C}$ ) will stand for a positive constant just depending on $\Omega, \mathcal{O}$ and $\delta_{0}$ (resp. on $\Omega, \mathcal{O}, \delta_{0}$ and $\left.\|\hat{u}\|_{\hat{Z}},\|\hat{b}\|_{W^{1, \infty}(0, T)},\|\hat{r}\|_{L^{\infty}(0, T)}\right)$. 


\subsection{A. Carleman estimate for the heat equation}

Let us apply the curl operator to the equation satisfied by $(\varphi, \pi)$ :

$$
-(\nabla \times \varphi)_{t}-(\hat{u} \cdot \nabla)(\nabla \times \varphi)-\mu \Delta(\nabla \times \varphi)=L(\hat{u}, \varphi) \quad \text { in } \widehat{\Omega}_{F}(t),
$$

where the right-hand side satisfies

$$
|L(\hat{u}, \varphi)| \leq C|\nabla \hat{u}||\nabla \varphi| \quad \text { in } \widehat{\Omega}_{F}(t) .
$$

Therefore, $\nabla \times \varphi$ satisfies a system of three heat equations. For this kind of system, Carleman inequalities are well-understood since [10]. Here, we are going to use an inequality which has been proved in [4, Section 2.1]. More precisely, we use the first inequality in $[4$, p. 2] by observing that, for the second term of the third line of that inequality, we have $e^{-2 s \mathcal{V}^{*}} \gamma^{2}=|\nabla w \tau|^{2}(\tau$ is the tangential vector field) in the notations of [4]. Using this for $\psi:=e^{-2 s \alpha} \nabla \times \varphi$, we can deduce

$$
\begin{aligned}
s^{3} \lambda^{4} \int_{0}^{T} \int_{\widehat{\Omega}_{F}(t)} \xi^{3}|\psi|^{2} d x d t+s \lambda^{2} \int_{0}^{T} \int_{\widehat{\Omega}_{F}(t)} \xi|\nabla \psi|^{2} d x d t \\
\quad+s^{3} \lambda^{3} \int_{0}^{T} \int_{\partial \widehat{\Omega}_{F}(t)}\left(\xi^{*}\right)^{3}|\psi|^{2} d \gamma d t+s \lambda \int_{0}^{T} \int_{\partial \widehat{\Omega}_{F}(t)} \xi^{*}|\nabla \psi n|^{2} d \gamma d t \\
\leq C\left(s^{3} \lambda^{4} \int_{0}^{T} \int_{\mathcal{O}_{0}} \xi^{3}|\psi|^{2} d x d t+s \lambda^{2} \int_{0}^{T} \int_{\mathcal{O}_{0}} \xi|\nabla \psi|^{2} d x d t\right. \\
\quad+s \lambda \int_{0}^{T} \int_{\partial \widehat{\Omega}_{F}(t)} \xi^{*}|\nabla \psi \tau|^{2} d \gamma d t+s \lambda^{2} \int_{0}^{T} \int_{\partial \widehat{\Omega}_{F}(t)} \xi^{*}|\nabla \psi n||\psi| d \gamma d t \\
\left.\quad+\int_{0}^{T} \int_{\partial \widehat{\Omega}_{F}(t)}|\nabla \psi n|\left|\psi_{t}+(\hat{u} \cdot \nabla) \psi\right| d \gamma d t+\int_{0}^{T} \int_{\widehat{\Omega}_{F}(t)} e^{-2 s \alpha}|\nabla \hat{u}|^{2}|\nabla \varphi|^{2} d x d t\right)
\end{aligned}
$$

for all $\lambda \geq \widehat{C}$ and all $s \geq C\left(T^{k}+T^{2 k}\right)$. Young's inequality applied to the fourth and fifth terms on the right-hand side of this inequality yields

$$
\begin{aligned}
& s^{3} \lambda^{4} \int_{0}^{T} \int_{\widehat{\Omega}_{F}(t)} \xi^{3}|\psi|^{2} d x d t+s \lambda^{2} \int_{0}^{T} \int_{\widehat{\Omega}_{F}(t)} \xi|\nabla \psi|^{2} d x d t \\
& \quad+s^{3} \lambda^{3} \int_{0}^{T} \int_{\partial \widehat{\Omega}_{F}(t)}\left(\xi^{*}\right)^{3}|\psi|^{2} d \gamma d t+s \lambda \int_{0}^{T} \int_{\partial \widehat{\Omega}_{F}(t)} \xi^{*}|\nabla \psi n|^{2} d \gamma d t \\
& \leq C\left(s^{3} \lambda^{4} \int_{0}^{T} \int_{\mathcal{O}_{0}} \xi^{3}|\psi|^{2} d x d t+s \lambda^{2} \int_{0}^{T} \int_{\mathcal{O}_{0}} \xi|\nabla \psi|^{2} d x d t\right. \\
&+s \lambda \int_{0}^{T} \int_{\partial \widehat{\Omega}_{F}(t)} \xi^{*}|\nabla \psi \tau|^{2} d \gamma d t+\int_{0}^{T} \int_{\partial \widehat{\Omega}_{F}(t)}\left(s \lambda \xi^{*}\right)^{-1}\left|\psi_{t}+(\hat{u} \cdot \nabla) \psi\right|^{2} d \gamma d t \\
&\left.+\int_{0}^{T} \int_{\widehat{\Omega}_{F}(t)} e^{-2 s \alpha}|\nabla \hat{u}|^{2}|\nabla \varphi|^{2} d x d t\right)
\end{aligned}
$$


for all $\lambda \geq \widehat{C}$ and all $s \geq C\left(T^{k}+T^{2 k}\right)$. We can estimate the term $(\hat{u} \cdot \nabla) \psi$ in the fourth integral of the right-hand side thanks to (12) (since $\hat{Z} \subset C^{0}\left(L^{\infty}\right)$ ):

$$
s^{-1} \lambda^{-1} \int_{0}^{T} \int_{\partial \widehat{\Omega}_{F}(t)}\left(\xi^{*}\right)^{-1}|(\hat{u} \cdot \nabla) \psi|^{2} d \gamma d t \leq \varepsilon s \lambda \int_{0}^{T} \int_{\partial \widehat{\Omega}_{F}(t)} \xi^{*}|\nabla \psi|^{2} d \gamma d t
$$

for $\varepsilon>0$ small enough provided that $\lambda \geq \widehat{C}_{\varepsilon}$ and $s \geq C_{\varepsilon} T^{2 k}$.

We come back to $\varphi$ now. Observe that the boundary term concerning $\psi_{t}$ in (20) can be bounded as follows:

$$
\begin{aligned}
& s^{-1} \lambda^{-1} \int_{0}^{T} \int_{\partial \widehat{\Omega}_{F}(t)}\left(\xi^{*}\right)^{-1}\left|\psi_{t}\right|^{2} d \gamma d t \\
& \leq 2\left(s^{-1} \lambda^{-1} \int_{0}^{T} \int_{\partial \widehat{\Omega}_{F}(t)} e^{-2 s \alpha^{*}}\left(\xi^{*}\right)^{-1}\left|\nabla \times \varphi_{t}\right|^{2} d \gamma d t\right. \\
&\left.\quad+s^{-1} \lambda^{-1} \int_{0}^{T} \int_{\partial \widehat{\Omega}_{F}(t)}\left|\partial_{t}\left(e^{-s \alpha^{*}}\right)\right|^{2}\left(\xi^{*}\right)^{-1}|\nabla \times \varphi|^{2} d \gamma d t\right) .
\end{aligned}
$$

Since $\left|\alpha_{t}^{*}\right| \leq \widehat{C}\left(T+T^{2}\right)\left(\xi^{*}\right)^{1+1 / k}$, the last term can be absorbed by the third integral on the left-hand side of (20) by taking $\lambda \geq \widehat{C}$ and $s \geq C\left(T^{2 k-1}+T^{2 k}\right)$. Thus, using also that $\nabla \beta \cdot \tau=0$ on $\partial \widehat{\Omega}_{S}(t)$ for the third term on the right-hand side of (20), we can rewrite estimate (20) in the following way:

$$
\begin{aligned}
& s^{3} \lambda^{4} \int_{0}^{T} \int_{\widehat{\Omega}_{F}(t)} e^{-2 s \alpha} \xi^{3}|\nabla \times \varphi|^{2} d x d t+s \lambda^{2} \int_{0}^{T} \int_{\widehat{\Omega}_{F}(t)} e^{-2 s \alpha} \xi|\nabla(\nabla \times \varphi)|^{2} d x d t \\
& \leq C\left(s^{3} \lambda^{4} \int_{0}^{T} \int_{\mathcal{O}_{0}} e^{-2 s \alpha} \xi^{3}|\nabla \times \varphi|^{2} d x d t+s \lambda^{2} \int_{0}^{T} \int_{\mathcal{O}_{0}} e^{-2 s \alpha} \xi|\nabla(\nabla \times \varphi)|^{2} d x d t\right. \\
& \quad+s \lambda \int_{0}^{T} \int_{\partial \widehat{\Omega}_{F}(t)} e^{-2 s \alpha^{*}} \xi^{*}|\nabla(\nabla \times \varphi) \tau|^{2} d \gamma d t \\
& \left.\quad+\int_{0}^{T} \int_{\partial \widehat{\Omega}_{F}(t)} e^{-2 s \alpha^{*}}\left(s \lambda \xi^{*}\right)^{-1}|\nabla \times \varphi|^{2} d \gamma d t+\int_{0}^{T} \int_{\widehat{\Omega}_{F}(t)} e^{-2 s \alpha}|\nabla \hat{u}|^{2}|\nabla \varphi|^{2} d x d t\right)
\end{aligned}
$$

for $\lambda \geq \widehat{C}$ and $s \geq C\left(T^{k}+T^{2 k}\right)$.

Let us obtain estimates on the second term on the right-hand side of (21). Let $\mathcal{O}_{1}$ be an open set with $\mathcal{O}_{0} \subset \subset \mathcal{O}_{1} \subset \subset \mathcal{O}_{2}$ and $\theta_{0} \in C_{c}^{2}\left(\mathcal{O}_{1}\right)$ be a positive function satisfying $\theta_{0}(x)=1$ for all $x \in \mathcal{O}_{0}$. We apply the curl operator to the first equation in (15):

$$
-(\nabla \times \varphi)_{t}-\nabla \times[(\hat{u} \cdot \nabla) \varphi]-\mu \Delta(\nabla \times \varphi)=0 \quad \text { in } \widehat{\Omega}_{F}(t) .
$$

Then we set $\rho(t, x):=s \lambda^{2} \theta_{0}(x) e^{-2 s \alpha(t, x)} \xi(t, x)$, we multiply the above equation by $\rho \nabla \times \varphi$ and we integrate by parts in $\mathcal{O}_{0}$ to obtain 


$$
\begin{gathered}
-\frac{1}{2} \frac{d}{d t} \int_{\mathcal{O}_{0}} \rho|\nabla \times \varphi|^{2} d x+\frac{1}{2} \int_{\mathcal{O}_{0}} \rho_{t}|\nabla \times \varphi|^{2} d x+\int_{\mathcal{O}_{0}} \rho[(\hat{u} \cdot \nabla)(\nabla \times \varphi)] \cdot(\nabla \times \varphi) d x \\
+\int_{\mathcal{O}_{0}}[(\nabla \rho) \times(\nabla \times \varphi)] \cdot[(\hat{u} \cdot \nabla) \varphi] d x+\int_{\mathcal{O}_{0}} \rho \Delta \varphi \cdot[(\hat{u} \cdot \nabla) \varphi] d x \\
\quad+\mu \int_{\mathcal{O}_{0}} \rho|\nabla(\nabla \times \varphi)|^{2} d x-\frac{\mu}{2} \int_{\mathcal{O}_{0}} \Delta \rho|\nabla \times \varphi|^{2} d x=0
\end{gathered}
$$

for $t \in(0, T)$. Next, we integrate between $t=0$ and $t=T$, and we use $\hat{u} \in \hat{Z} \subset C^{0}\left(L^{\infty}\right)$ and

to obtain

$$
\left|\rho_{t}\right|+|\Delta \rho| \leq C s^{3} \lambda^{4} \xi^{3} e^{-2 s \alpha} \quad \text { for } s \geq C\left(T^{k}+T^{2 k}\right) \text { and } \lambda \geq C,
$$

$s \lambda^{2} \int_{0}^{T} \int_{\mathcal{O}_{0}} e^{-2 s \alpha} \xi|\nabla(\nabla \times \varphi)|^{2} d x d t$

$\leq \widehat{C}\left(s^{3} \lambda^{4} \int_{0}^{T} \int_{\mathcal{O}_{1}} e^{-2 s \alpha} \xi^{3}|\nabla \times \varphi|^{2} d x d t+s \lambda^{2} \int_{0}^{T} \int_{\mathcal{O}_{1}} e^{-2 s \alpha} \xi^{-1}|\nabla \varphi|^{2} d x d t\right)$

for $\lambda \geq \widehat{C}$ and $s \geq C\left(T^{k}+T^{2 k}\right)$. Observe that the second term on the right-hand side can be bounded by the last one in (21) by taking $s \geq C T^{2 k}$. Next, we estimate the local term with $\nabla \times \varphi$. In order to do this, let $\theta_{1} \in C_{c}^{2}\left(\mathcal{O}_{2}\right)$ be a positive function satisfying $\theta_{1}(x)=1$ for all $x \in \mathcal{O}_{1}$. Then integrating by parts in

$s^{3} \lambda^{4} \int_{0}^{T} \int_{\mathcal{O}_{2}} \theta_{1} e^{-2 s \alpha} \xi^{3}(\nabla \times \varphi) \cdot(\nabla \times \varphi) d x d t+s \lambda^{2} \int_{0}^{T} \int_{\mathcal{O}_{2}} \theta_{1} e^{-2 s \alpha} \xi(\nabla \varphi) \cdot(\nabla \varphi) d x d t$ we can prove that

$$
\begin{array}{r}
s^{3} \lambda^{4} \int_{0}^{T} \int_{\mathcal{O}_{1}} e^{-2 s \alpha} \xi^{3}|\nabla \times \varphi|^{2} d x d t+s \lambda^{2} \int_{0}^{T} \int_{\mathcal{O}_{1}} e^{-2 s \alpha} \xi|\nabla \varphi|^{2} d x d t \\
\leq C_{\varepsilon} s^{5} \lambda^{6} \int_{0}^{T} \int_{\mathcal{O}_{2}} e^{-2 s \alpha} \xi^{5}|\varphi|^{2} d x d t+\varepsilon\left(s \lambda^{2} \int_{0}^{T} \int_{\mathcal{O}_{2}} e^{-2 s \alpha} \xi|\nabla(\nabla \times \varphi)|^{2} d x d t\right. \\
\left.+s^{3} \lambda^{4} \int_{0}^{T} \int_{\mathcal{O}_{2}} e^{-2 s \alpha} \xi^{3}|\nabla \times \varphi|^{2} d x d t\right)
\end{array}
$$

for $\lambda \geq C$ and $s \geq C T^{2 k}$. Combining this with (21) and (23), we obtain

$$
\begin{aligned}
s^{3} \lambda^{4} & \int_{0}^{T} \int_{\widehat{\Omega}_{F}(t)} e^{-2 s \alpha} \xi^{3}|\nabla \times \varphi|^{2} d x d t+s \lambda^{2} \int_{0}^{T} \int_{\widehat{\Omega}_{F}(t)} e^{-2 s \alpha} \xi|\nabla(\nabla \times \varphi)|^{2} d x d t \\
\leq & C\left(s^{5} \lambda^{6} \int_{0}^{T} \int_{\mathcal{O}_{2}} e^{-2 s \alpha} \xi|\varphi|^{2} d x d t+s \lambda \int_{0}^{T} \int_{\partial \widehat{\Omega}_{F}(t)} e^{-2 s \alpha^{*}} \xi^{*}|\nabla(\nabla \times \varphi) \tau|^{2} d \gamma d t\right. \\
& +s^{-1} \lambda^{-1} \int_{0}^{T} \int_{\partial \widehat{\Omega}_{F}(t)} e^{-2 s \alpha^{*}}\left(\xi^{*}\right)^{-1}\left|\nabla \times \varphi_{t}\right|^{2} d \gamma d t \\
& \left.+\int_{0}^{T} \int_{\widehat{\Omega}_{F}(t)} e^{-2 s \alpha}|\nabla \hat{u}|^{2}|\nabla \varphi|^{2} d x d t\right)
\end{aligned}
$$

for $\lambda \geq \widehat{C}$ and $s \geq C\left(T^{k}+T^{2 k}\right)$. 


\section{B. Elliptic estimates}

Since $\nabla \cdot \varphi=0$, observe that $\varphi$ satisfies the following boundary-value problem:

$$
\begin{cases}\Delta \varphi=-\nabla \times(\nabla \times \varphi):=f_{0} & \text { in } \widehat{\Omega}_{F}(t), \\ \varphi=(\dot{a}+\omega \times(x-\hat{b})) 1_{\partial \widehat{\Omega}_{S}(t)}:=g_{0} & \text { on } \partial \widehat{\Omega}_{F}(t) .\end{cases}
$$

- Applying classical elliptic estimates, we have

$$
\begin{aligned}
\|\varphi\|_{H^{1}\left(\widehat{\Omega}_{F}(t)\right)} & \leq \widehat{C}\left(\left\|f_{0}\right\|_{H^{-1}\left(\widehat{\Omega}_{F}(t)\right)}+\left\|g_{0}\right\|_{H^{1 / 2}\left(\partial \widehat{\Omega}_{F}(t)\right)}\right) \\
& \leq \widehat{C}\left(\|\nabla \times \varphi\|_{L^{2}\left(\widehat{\Omega}_{F}(t)\right)}+|\dot{a}|+|\omega|\right),
\end{aligned}
$$

which directly leads to

$$
\begin{aligned}
s^{3} \lambda^{4} \int_{0}^{T} \int_{\widehat{\Omega}_{F}(t)} e^{-2 s \alpha^{*}}\left(\xi^{*}\right)^{3}|\nabla \varphi|^{2} d x d t & \leq \widehat{C}\left(s^{3} \lambda^{4} \int_{0}^{T} \int_{\widehat{\Omega}_{F}(t)} e^{-2 s \alpha} \xi^{3}|\nabla \times \varphi|^{2} d x d t\right. \\
& \left.+s^{3} \lambda^{4} \int_{0}^{T} e^{-2 s \alpha^{*}}\left(\xi^{*}\right)^{3}\left(|\dot{a}|^{2}+|\omega|^{2}\right) d t\right) .
\end{aligned}
$$

- We now apply the classical elliptic Carleman estimate which can be proved as in [10]:

$$
\begin{aligned}
\kappa^{4} \lambda^{6} \int_{\widehat{\Omega}_{F}(t)} \exp \left\{2 \kappa e^{\lambda \beta}\right\} e^{4 \lambda \beta}|\varphi|^{2} d x+\kappa^{2} \lambda^{4} \int_{\widehat{\Omega}_{F}(t)} \exp \left\{2 \kappa e^{\lambda \beta}\right\} e^{2 \lambda \beta}|\nabla \varphi|^{2} d x & +\kappa^{4} \lambda^{5} \int_{\partial \widehat{\Omega}_{F}(t)} e^{2 \kappa}|\varphi|^{2} d \gamma \\
\leq & C\left(\kappa^{4} \lambda^{6} \int_{\mathcal{O}_{2}} \exp \left\{2 \kappa e^{\lambda \beta}\right\} e^{4 \lambda \beta}|\varphi|^{2} d x+\kappa \lambda^{2} \int_{\widehat{\Omega}_{F}(t)} \exp \left\{2 \kappa e^{\lambda \beta}\right\} e^{\lambda \beta}\left|f_{0}\right|^{2} d x\right. \\
& \left.+\kappa^{2} \lambda^{3} e^{2 \kappa} \int_{\partial \widehat{\Omega}_{F}(t)}\left|\partial_{\kappa} g_{0}\right|^{2} d \gamma\right)
\end{aligned}
$$

for any $\kappa \geq \widehat{C}$ and any $\lambda \geq \widehat{C}$. Combining this with $H^{2}$ elliptic estimates, we deduce that

$$
\begin{aligned}
& \kappa^{4} \lambda^{6} \int_{\widehat{\Omega}_{F}(t)} \exp \left\{2 \kappa e^{\lambda \beta}\right\} e^{4 \lambda \beta}|\varphi|^{2} d x+\kappa^{2} \lambda^{4} \int_{\widehat{\Omega}_{F}(t)} \exp \left\{2 \kappa e^{\lambda \beta}\right\} e^{2 \lambda \beta}|\nabla \varphi|^{2} d x \\
& \quad+\kappa^{4} \lambda^{5} \int_{\partial \widehat{\Omega}_{F}(t)} e^{2 \kappa}|\varphi|^{2} d \gamma+\lambda^{2} \int_{\widehat{\Omega}_{F}(t)} \exp \left\{2 \kappa e^{\lambda \beta}\right\}\left|D^{2} \varphi\right|^{2} d x \\
& \leq C\left(\kappa^{4} \lambda^{6} \int_{\mathcal{O}_{2}} \exp \left\{2 \kappa e^{\lambda \beta}\right\} e^{4 \lambda \beta}|\varphi|^{2} d x\right. \\
&\left.+\kappa \lambda^{2} \int_{\widehat{\Omega}_{F}(t)} \exp \left\{2 \kappa e^{\lambda \beta}\right\} e^{\lambda \beta}|\Delta \varphi|^{2} d x+\kappa^{2} \lambda^{3} e^{2 \kappa}\left(|\dot{a}|^{2}+|\omega|^{2}\right)\right)
\end{aligned}
$$

for any $\kappa \geq \widehat{C}$ and any $\lambda \geq \widehat{C}$, where we have used

$$
\|\varphi\|_{H^{3 / 2}\left(\partial \widehat{\Omega}_{F}(t)\right)} \leq \widehat{C}(|\dot{a}|+|\omega|) .
$$


We set $\kappa:=s e^{2 k \lambda M} /\left(t^{k}(T-t)^{k}\right)$ and we multiply the previous inequality by

$$
\exp \left\{-2 s \frac{e^{(2 k+2) \lambda M}}{t^{k}(T-t)^{k}}\right\}
$$

This yields

$$
\begin{aligned}
\lambda^{2} \int_{0}^{T} \int_{\widehat{\Omega}_{F}(t)} e^{-2 s \alpha}\left(s^{4} \lambda^{4} \xi^{4}|\varphi|^{2}+s^{2} \lambda^{2} \xi^{2}|\nabla \varphi|^{2}\right. & \left.+\left|D^{2} \varphi\right|^{2}\right) d x d t \\
& +s^{4} \lambda^{5} \int_{0}^{T} \int_{\partial \widehat{\Omega}_{F}(t)} e^{-2 s \alpha^{*}}\left(\xi^{*}\right)^{4}|\varphi|^{2} d \gamma d t \\
\leq & C\left(s^{4} \lambda^{6} \int_{0}^{T} \int_{\mathcal{O}_{2}} e^{-2 s \alpha} \xi^{4}|\varphi|^{2} d x d t+s \lambda^{2} \int_{0}^{T} \int_{\widehat{\Omega}_{F}(t)} e^{-2 s \alpha} \xi|\Delta \varphi|^{2} d x d t\right. \\
& \left.+s^{2} \lambda^{3} \int_{0}^{T} e^{-2 s \alpha^{*}}\left(\xi^{*}\right)^{2}\left(|\dot{a}|^{2}+|\omega|^{2}\right) d t\right)
\end{aligned}
$$

for $\lambda \geq \widehat{C}$ and $s \geq \widehat{C}\left(T^{k}+T^{2 k}\right)$. Observe that the terms $|\nabla \varphi|^{2}$ and $\left|D^{2} \varphi\right|^{2}$ on the left-hand side of (26) allow the last term in (24) to be absorbed by taking $\lambda \geq \widehat{C}$ and $s \geq C T^{2 k}$ and using $\hat{u} \in C^{0}\left(W^{1,3}\right)$. Combining this with (25) and (24), we obtain

$$
\begin{aligned}
& s^{4} \lambda^{6} \int_{0}^{T} \int_{\widehat{\Omega}_{F}(t)} e^{-2 s \alpha} \xi^{4}|\varphi|^{2} d x d t+s^{3} \lambda^{4} \int_{0}^{T} \int_{\widehat{\Omega}_{F}(t)} e^{-2 s \alpha^{*}}\left(\xi^{*}\right)^{3}|\nabla \varphi|^{2} d x d t \\
& \quad+s^{4} \lambda^{5} \int_{0}^{T} \int_{\partial \widehat{\Omega}_{F}(t)} e^{-2 s \alpha^{*}}\left(\xi^{*}\right)^{4}|\varphi|^{2} d \gamma d t \\
& \leq \quad C\left(s^{5} \lambda^{6} \int_{0}^{T} \int_{\mathcal{O}_{2}} e^{-2 s \alpha} \xi^{5}|\varphi|^{2} d x d t+s \lambda \int_{0}^{T} \int_{\partial \widehat{\Omega}_{F}(t)} e^{-2 s \alpha^{*} \xi^{*}|\nabla(\nabla \times \varphi) \tau|^{2} d \gamma d t}\right. \\
& \quad+s^{-1} \lambda^{-1} \int_{0}^{T} \int_{\partial \widehat{\Omega}_{F}(t)} e^{-2 s \alpha^{*}}\left(\xi^{*}\right)^{-1}\left|\nabla \times \varphi_{t}\right|^{2} d \gamma d t \\
& \left.\quad+s^{3} \lambda^{4} \int_{0}^{T} e^{-2 s \alpha^{*}}\left(\xi^{*}\right)^{3}\left(|\dot{a}|^{2}+|\omega|^{2}\right) d t\right)
\end{aligned}
$$

for $\lambda \geq \widehat{C}$ and $s \geq \widehat{C}\left(T^{k}+T^{2 k}\right)$.

We notice that

$$
\int_{\partial \widehat{\Omega}_{S}(t)}|\varphi|^{2} d \gamma \geq \widehat{C}\left(|\dot{a}|^{2}+|\omega|^{2}\right) .
$$

The proof of this inequality is given in [3, Lemma 1, Section 4.1]. This allows the last term on the right-hand side of (27) to be absorbed thanks to $s \geq \widehat{C} T^{2 k}$. For the moment, 
we have

$$
\begin{gathered}
s^{4} \lambda^{6} \int_{0}^{T} \int_{\widehat{\Omega}_{F}(t)} e^{-2 s \alpha} \xi^{4}|\varphi|^{2} d x d t+s^{3} \lambda^{4} \int_{0}^{T} \int_{\widehat{\Omega}_{F}(t)} e^{-2 s \alpha^{*}}\left(\xi^{*}\right)^{3}|\nabla \varphi|^{2} d x d t \\
+s^{4} \lambda^{5} \int_{0}^{T} e^{-2 s \alpha^{*}}\left(\xi^{*}\right)^{4}\left(|\dot{a}|^{2}+|\omega|^{2}\right) d t \\
\leq C\left(s^{5} \lambda^{6} \int_{0}^{T} \int_{\mathcal{O}_{2}} e^{-2 s \alpha} \xi^{5}|\varphi|^{2} d x d t+s \lambda \int_{0}^{T} \int_{\partial \widehat{\Omega}_{F}(t)} e^{-2 s \alpha^{*}} \xi^{*}|\nabla(\nabla \times \varphi) \tau|^{2} d \gamma d t\right. \\
\left.\quad+s^{-1} \lambda^{-1} \int_{0}^{T} \int_{\partial \widehat{\Omega}_{F}(t)} e^{-2 s \alpha^{*}}\left(\xi^{*}\right)^{-1}\left|\nabla \times \varphi_{t}\right|^{2} d \gamma d t\right)
\end{gathered}
$$

for $\lambda \geq \widehat{C}$ and $s \geq \widehat{C}\left(T^{k}+T^{2 k}\right)$.

The rest of the proof is dedicated to the estimate of the two boundary terms

$$
\begin{aligned}
& B_{1}:=s \lambda \int_{0}^{T} \int_{\partial \widehat{\Omega}_{F}(t)} e^{-2 s \alpha^{*} \xi^{*}|\nabla(\nabla \times \varphi) \tau|^{2} d \gamma d t,} \\
& B_{2}:=s^{-1} \lambda^{-1} \int_{0}^{T} \int_{\partial \widehat{\Omega}_{F}(t)} e^{-2 s \alpha^{*}}\left(\xi^{*}\right)^{-1}\left|\nabla \times \varphi_{t}\right|^{2} d \gamma d t .
\end{aligned}
$$

\section{Estimate of $B_{1}$}

Let us define, on $(0, T)$,

$$
\theta_{1}:=s^{1 / 2} \lambda^{1 / 2} e^{-s \alpha^{*}}\left(\xi^{*}\right)^{1 / 2},
$$

and set $\left(\varphi^{*}, \pi^{*}, \dot{a}^{*}, \omega^{*}\right):=\theta_{1}(\varphi, \pi, \dot{a}, \omega)$ together with $a^{*}(T)=0$. These functions satisfy

$$
\begin{cases}\left.-\varphi_{t}^{*}(t, x)-(\hat{u} \cdot \nabla) \varphi^{*}\right)(t, x)-\nabla \cdot \sigma\left(\varphi^{*}, \pi^{*}\right)(t, x)=-\dot{\theta}_{1} \varphi, & x \in \widehat{\Omega}_{F}(t), \\ \nabla \cdot \varphi^{*}(t, x)=0, & x \in \widehat{\Omega}_{F}(t), \\ \varphi^{*}(t, x)=0, & x \in \partial \Omega, \\ \varphi^{*}(t, x)=\dot{a}^{*}(t)+\omega^{*}(t) \times(x-\hat{b}(t)), & x \in \partial \widehat{\Omega}_{S}(t), \\ m \ddot{a^{*}}(t)=-\int_{\partial \widehat{\Omega}_{S}(t)}\left(\sigma\left(\varphi^{*}, \pi^{*}\right) n\right)(t, x) d \gamma+m \dot{\theta}_{1} \dot{a}, & \\ \frac{d}{d t}\left(\hat{J} \omega^{*}\right)(t)=\left((\hat{J} \hat{r}) \times \omega^{*}\right)(t)-\int_{\partial \widehat{\Omega}_{S}(t)}(x-\hat{b}(t)) \times\left(\sigma\left(\varphi^{*}, \pi^{*}\right) n\right)(t, x) d \gamma+\hat{J} \dot{\theta}_{1} \omega, \\ \varphi_{\mid t=T}^{*}=0 \quad \text { in } \widehat{\Omega}_{F}(T), \quad a^{*}(T)=\dot{a}^{*}(T)=0, \omega^{*}(T)=0 .\end{cases}
$$

Here, we apply Corollary 9 (stated in the Appendix) with $k_{0}=13 / 9$ and we deduce the existence of a constant $\widehat{C}$ such that

$$
\begin{aligned}
& \left\|\theta_{1} \varphi\right\|_{L^{2}\left(H^{23 / 9}\right)}+\left\|\theta_{1} \varphi\right\|_{H^{1}\left(H^{5 / 9}\right)}+\left\|\theta_{1} \dot{a}\right\|_{H^{23 / 18}(0, T)}+\left\|\theta_{1} \omega\right\|_{H^{23 / 18}(0, T)} \\
& \leq \widehat{C}\left(\left\|\dot{\theta}_{1} \varphi\right\|_{L^{2}\left(H^{5 / 9}\right)}+\left\|\dot{\theta}_{1} \varphi\right\|_{H^{5 / 18}\left(L^{2}\right)}+\left\|\dot{\theta}_{1} \dot{a}\right\|_{H^{5 / 18}}+\left\|\dot{\theta}_{1} \omega\right\|_{\left.H^{5 / 18}\right)} .\right.
\end{aligned}
$$


Since 23/9 > 5/2, $B_{1} \leq \widehat{C}\left\|\theta_{1} \varphi\right\|_{L^{2}\left(H^{23 / 9}\right)}^{2}$, it suffices to estimate all four terms on the right-hand side of (30).

C.1. Estimate of $\left\|\dot{\theta}_{1} \varphi\right\|_{L^{2}\left(H^{5 / 9}\right)}$. By an interpolation argument, we have

$$
\|\varphi\|_{H^{5 / 9}\left(\widehat{\Omega}_{F}(t)\right)} \leq \widehat{C}\|\varphi\|_{L^{2}\left(\widehat{\Omega}_{F}(t)\right)}^{4 / 9}\|\varphi\|_{H^{1}\left(\widehat{\Omega}_{F}(t)\right)}^{5 / 9} .
$$

Multiplying this inequality by $\dot{\theta}_{1}$, we obtain

$$
\begin{aligned}
& \dot{\theta}_{1}\|\varphi\|_{H^{5 / 9}\left(\widehat{\Omega}_{F}(t)\right)} \\
& \quad \leq \widehat{C} s^{2 / 9}\left(\xi^{*}\right)^{2 / 9-4 /(9 k)}\left(\dot{\theta}_{1}\right)^{4 / 9}\|\varphi\|_{L^{2}\left(\widehat{\Omega}_{F}(t)\right)}^{4 / 9} s^{-2 / 9}\left(\xi^{*}\right)^{-2 / 9+4 /(9 k)}\left(\dot{\theta}_{1}\right)^{5 / 9}\|\varphi\|_{H^{1}\left(\widehat{\Omega}_{F}(t)\right)}^{5 / 9} .
\end{aligned}
$$

Applying now Young's inequality, we get

$$
\begin{aligned}
\dot{\theta}_{1}\|\varphi\|_{H^{5 / 9}\left(\widehat{\Omega}_{F}(t)\right)} \leq & \varepsilon s^{1 / 2}\left(\xi^{*}\right)^{1 / 2-1 / k} \dot{\theta}_{1}\|\varphi\|_{L^{2}\left(\widehat{\Omega}_{F}(t)\right)} \\
& +\widehat{C}_{\varepsilon} s^{-2 / 5}\left(\xi^{*}\right)^{-2 / 5+4 /(5 k)} \dot{\theta}_{1}\|\varphi\|_{H^{1}\left(\widehat{\Omega}_{F}(t)\right)} .
\end{aligned}
$$

Observe that

$$
\left|\dot{\theta}_{1}\right| \leq s^{3 / 2} \lambda^{1 / 2} e^{-s \alpha^{*}}\left(\xi^{*}\right)^{3 / 2+1 / k},
$$

with $s \geq \widehat{C}\left(T^{k}+T^{2 k}\right)$.

Integrating inequality (31) in time, we obtain

$$
\begin{aligned}
\left\|\dot{\theta}_{1} \varphi\right\|_{L^{2}\left(H^{5 / 9}\right)}^{2} \leq & \varepsilon s^{4} \lambda \int_{0}^{T} \int_{\widehat{\Omega}_{F}(t)} e^{-2 s \alpha^{*}}\left(\xi^{*}\right)^{4}|\varphi|^{2} d x d t \\
& +C_{\varepsilon} s^{11 / 5} \lambda \int_{0}^{T} \int_{\widehat{\Omega}_{F}(t)} e^{-2 s \alpha^{*}}\left(\xi^{*}\right)^{11 / 5+18 /(5 k)}|\nabla \varphi|^{2} d x d t .
\end{aligned}
$$

These two terms can be absorbed by the left-hand side of the Carleman inequality (28) provided that $k \geq 9, s \geq C\left(T^{k}+T^{2 k}\right)$ and $\lambda \geq 1$.

C.2. Estimate of $\left\|\dot{\theta}_{1} \varphi\right\|_{H^{5 / 8}\left(L^{2}\right)}$. Observe that

$$
\left\|\dot{\theta}_{1} \varphi\right\|_{L^{2}\left(L^{2}\right)}^{2} \leq \widehat{C} s^{3} \lambda \int_{0}^{T} \int_{\widehat{\Omega}_{F}(t)} e^{-2 s \alpha^{*}}\left(\xi^{*}\right)^{3+2 / k}|\varphi|^{2} d x d t
$$

and

$$
\begin{aligned}
\left\|\dot{\theta}_{1} \varphi\right\|_{H^{1}\left(L^{2}\right)}^{2} \leq & \widehat{C}\left(s^{3} \lambda \int_{0}^{T} \int_{\widehat{\Omega}_{F}(t)} e^{-2 s \alpha^{*}}\left(\xi^{*}\right)^{3+2 / k}\left|\varphi_{t}\right|^{2} d x d t\right. \\
& \left.+s^{5} \lambda \int_{0}^{T} \int_{\widehat{\Omega}_{F}(t)} e^{-2 s \alpha^{*}}\left(\xi^{*}\right)^{5+4 / k}|\varphi|^{2} d x d t\right) .
\end{aligned}
$$


By an interpolation argument due to [18], we get

$\left\|\dot{\theta}_{1} \varphi\right\|_{H^{5 / 18}\left(L^{2}\right)}^{2}$

$$
\begin{aligned}
\leq & \widehat{C} \lambda\left(\int_{0}^{T} \int_{\widehat{\Omega}_{F}(t)} e^{-2 s \alpha^{*}}\left(s^{3}\left(\xi^{*}\right)^{3+2 / k}|\varphi|^{2}\right)^{13 / 18}\left(s^{3}\left(\xi^{*}\right)^{3+2 / k}\left|\varphi_{t}\right|^{2}\right)^{5 / 18} d x d t\right. \\
& \left.+\int_{0}^{T} \int_{\widehat{\Omega}_{F}(t)} e^{-2 s \alpha^{*}}\left(s^{3}\left(\xi^{*}\right)^{3+2 / k}|\varphi|^{2}\right)^{13 / 18}\left(s^{5}\left(\xi^{*}\right)^{5+4 / k}|\varphi|^{2}\right)^{5 / 18} d x d t\right) \\
= & \widehat{C} \lambda\left(\int_{0}^{T} \int_{\widehat{\Omega}_{F}(t)} e^{-2 s \alpha^{*}}\left(s^{4}\left(\xi^{*}\right)^{4}|\varphi|^{2}\right)^{13 / 18}\left(s^{2 / 5}\left(\xi^{*}\right)^{2 / 5+36 /(5 k)}\left|\varphi_{t}\right|^{2}\right)^{5 / 18} d x d t\right. \\
& \left.+\int_{0}^{T} \int_{\widehat{\Omega}_{F}(t)} e^{-2 s \alpha^{*}}\left(s^{4}\left(\xi^{*}\right)^{4}|\varphi|^{2}\right)^{13 / 18}\left(s^{12 / 5}\left(\xi^{*}\right)^{12 / 5+46 /(5 k)}|\varphi|^{2}\right)^{5 / 18} d x d t\right) .
\end{aligned}
$$

In these two integrals, we apply Young's inequality with parameters $18 / 13$ and $18 / 5$ to find

$$
\begin{aligned}
& \left\|\dot{\theta}_{1} \varphi\right\|_{H^{5 / 18}\left(L^{2}\right)}^{2} \leq \widehat{C} \lambda s^{4} \int_{0}^{T} \int_{\widehat{\Omega}_{F}(t)} e^{-2 s \alpha^{*}}\left(\xi^{*}\right)^{4}|\varphi|^{2} d x d t \\
& +\widehat{C} \lambda s^{2 / 5} \int_{0}^{T} \int_{\widehat{\Omega}_{F}(t)} e^{-2 s \alpha^{*}}\left(\xi^{*}\right)^{2 / 5+36 /(5 k)}\left|\varphi_{t}\right|^{2} d x d t \\
& +\widehat{C} \lambda s^{12 / 5} \int_{0}^{T} \int_{\widehat{\Omega}_{F}(t)} e^{-2 s \alpha^{*}}\left(\xi^{*}\right)^{12 / 5+46 /(5 k)}|\varphi|^{2} d x d t .
\end{aligned}
$$

The first and third integrals can be absorbed by the left-hand side of (28) on taking $\lambda \geq \widehat{C}, s \geq C\left(T^{k}+T^{2 k}\right)$ and $k \geq 23 / 2$, while the second integral is absorbed by the second term on the left-hand side of (30) (squared) if we take $\lambda \geq \widehat{C}, s \geq C\left(T^{k}+T^{2 k}\right.$ ) and $k \geq 24$.

C.3. Estimate of $\left\|\dot{\theta}_{1} \dot{a}\right\|_{H^{5 / 18}}$. We have

$$
\left\|\dot{\theta}_{1} \dot{a}\right\|_{L^{2}(0, T)}^{2} \leq \widehat{C} s^{3} \lambda \int_{0}^{T} e^{-2 s \alpha^{*}}\left(\xi^{*}\right)^{3+2 / k}|\dot{a}|^{2} d t
$$

and

$\left\|\dot{\theta}_{1} \dot{a}\right\|_{H^{1}(0, T)}^{2} \leq \widehat{C}\left(s^{3} \lambda \int_{0}^{T} e^{-2 s \alpha^{*}}\left(\xi^{*}\right)^{3+2 / k}|\ddot{a}|^{2} d t+s^{5} \lambda \int_{0}^{T} e^{-2 s \alpha^{*}}\left(\xi^{*}\right)^{5+4 / k}|\dot{a}|^{2} d t\right)$.

Using again an interpolation argument from [18], we get

$$
\begin{aligned}
\left\|\dot{\theta}_{1} \dot{a}\right\|_{H^{5 / 18}(0, T)}^{2} \leq & \widehat{C} \lambda\left(\int_{0}^{T} e^{-2 s \alpha^{*}}\left(s^{3}\left(\xi^{*}\right)^{3+2 / k}|\dot{a}|^{2}\right)^{13 / 18}\left(s^{3}\left(\xi^{*}\right)^{3+2 / k}|\ddot{a}|^{2}\right)^{5 / 18} d t\right. \\
& \left.+\int_{0}^{T} e^{-2 s \alpha^{*}}\left(s^{3}\left(\xi^{*}\right)^{3+2 / k}|\dot{a}|^{2}\right)^{13 / 18}\left(s^{5}\left(\xi^{*}\right)^{5+4 / k}|\dot{a}|^{2}\right)^{5 / 18} d t\right) .
\end{aligned}
$$


We apply Young's inequality with parameters $18 / 13$ and $18 / 5$ to find

$$
\begin{aligned}
& \left\|\dot{\theta}_{1} \dot{a}\right\|_{H^{5 / 18}(0, T)}^{2} \leq \widehat{C} \lambda s^{32 / 9} \int_{0}^{T} e^{-2 s \alpha^{*}}\left(\xi^{*}\right)^{32 / 9+23 /(9 k)}|\dot{a}|^{2} d t \\
& \quad+\widehat{C} \lambda s^{2 / 5} \int_{0}^{T} e^{-2 s \alpha^{*}}\left(\xi^{*}\right)^{2 / 5+36 /(5 k)}|\ddot{a}|^{2} d t+\widehat{C} \lambda s^{4} \int_{0}^{T} e^{-2 s \alpha^{*}}\left(\xi^{*}\right)^{4}|\dot{a}|^{2} d t .
\end{aligned}
$$

The first and third integrals can be absorbed by the left-hand side of (28) taking $\lambda \geq \widehat{C}$, $s \geq C\left(T^{k}+T^{2 k}\right)$ and $k \geq 23 / 4$ while the second integral can be absorbed by the third term on the left-hand side of (30) provided that $\lambda \geq \widehat{C}, s \geq C\left(T^{k}+T^{2 k}\right)$ and $k \geq 12$.

C.4. Estimate of $\left\|\dot{\theta}_{1} \omega\right\|_{H^{5 / 18}}$. In order to estimate this term, we proceed exactly as in Step C.3.

To conclude Step C, we put together Steps C.1-C.4 to obtain

$$
\begin{aligned}
B_{1} \leq & \varepsilon\left(s^{4} \lambda^{6} \int_{0}^{T} \int_{\widehat{\Omega}_{F}(t)} e^{-2 s \alpha} \xi^{4}|\varphi|^{2} d x d t+s^{3} \lambda^{4} \int_{0}^{T} \int_{\widehat{\Omega}_{F}(t)} e^{-2 s \alpha^{*}}\left(\xi^{*}\right)^{3}|\nabla \varphi|^{2} d x d t\right. \\
& \left.+s^{4} \lambda^{5} \int_{0}^{T} e^{-2 s \alpha^{*}}\left(\xi^{*}\right)^{4}\left(|\dot{a}|^{2}+|\omega|^{2}\right) d t\right)
\end{aligned}
$$

for $\lambda \geq \widehat{C}_{\varepsilon}$ and $s \geq \widehat{C}_{\varepsilon}\left(T^{k}+T^{2 k}\right)$ for $k \geq 24$.

\section{Estimate of $B_{2}$}

Let us define, on $[0, T]$,

$$
\theta_{2}:=s^{-1 / 2} \lambda^{-1 / 2} e^{-s \alpha^{*}}\left(\xi^{*}\right)^{-1 / 2} .
$$

Then $\theta_{2}(\varphi, \pi, \dot{a}, \omega)$ satisfies system (29) with $\theta_{1}$ replaced by $\theta_{2}$. We notice that

$$
\left|B_{2}\right| \leq C\left\|\theta_{2} \varphi_{t}\right\|_{L^{2}\left(H^{14 / 9}\right)}^{2},
$$

since $14 / 9>3 / 2$.

Let us apply Corollary 9 for $k_{0}=4 / 9$. For our system, the compatibility condition (71) is satisfied since, thanks to the weight function $\theta_{2}$, all the initial conditions are equal to zero. This yields, in particular,

$$
\left\|\theta_{2} \varphi_{t}\right\|_{L^{2}\left(H^{14 / 9}\right)} \leq \widehat{C}\left(\left\|\dot{\theta}_{2} \varphi\right\|_{L^{2}\left(H^{14 / 9}\right) \cap H^{7 / 9}\left(L^{2}\right)}+\left\|\dot{\theta}_{2} \dot{a}\right\|_{H^{7 / 9}(0, T)}+\left\|\dot{\theta}_{2} \omega\right\|_{H^{7 / 9}(0, T)}\right) .
$$

Observe that

$$
\begin{aligned}
\left\|\dot{\theta}_{2} \varphi\right\|_{L^{2}\left(H^{14 / 9}\right) \cap H^{7 / 9}\left(L^{2}\right)}+\left\|\dot{\theta}_{2} \dot{a}\right\|_{H^{7 / 9}(0, T)}+\left\|\dot{\theta}_{2} \omega\right\|_{H^{7 / 9}(0, T)} \\
\quad \leq C\left(\left\|\dot{\theta}_{2} \varphi\right\|_{\hat{Y}_{0}}+\left\|\dot{\theta}_{2} \dot{a}\right\|_{H^{1}(0, T)}+\left\|\dot{\theta}_{2} \omega\right\|_{H^{1}(0, T)}\right) .
\end{aligned}
$$


Applying now Proposition 7 to $\dot{\theta}_{2}(\varphi, \pi, \dot{a}, \omega)$, we deduce

$$
\left\|\theta_{2} \varphi_{t}\right\|_{L^{2}\left(H^{14 / 9}\right)} \leq \widehat{C}\left(\left\|\ddot{\theta}_{2} \varphi\right\|_{L^{2}\left(L^{2}\right)}+\left\|\ddot{\theta}_{2} \dot{a}\right\|_{L^{2}(0, T)}+\left\|\ddot{\theta}_{2} \omega\right\|_{L^{2}(0, T)}\right) .
$$

Using the definition of the weight functions (see (16)), we obtain

$$
\left|\ddot{\theta}_{2}\right| \leq \widehat{C}\left(s \xi^{*}\right)^{3 / 2+2 / k} \lambda^{-1 / 2} e^{-s \alpha^{*}} \quad \text { for } s \geq \widehat{C}\left(T^{k}+T^{2 k}\right) .
$$

This readily implies that the first (resp. second and third) norm on the right-hand side of (34) is absorbed by the first (resp. third) integral on the left-hand side of (28) provided that $\lambda \geq \widehat{C}, s \geq \widehat{C}\left(T^{k}+T^{2 k}\right)$ and $k \geq 4$.

Consequently, we have proved that

$$
B_{2} \leq \varepsilon\left(s^{4} \lambda^{6} \int_{0}^{T} \int_{\widehat{\Omega}_{F}(t)} e^{-2 s \alpha} \xi^{4}|\varphi|^{2} d x d t+s^{4} \lambda^{5} \int_{0}^{T} e^{-2 s \alpha^{*}}\left(\xi^{*}\right)^{4}\left(|\dot{a}|^{2}+|\omega|^{2}\right) d t\right)
$$

for $\lambda \geq \widehat{C}_{\varepsilon}$ and $s \geq \widehat{C}_{\varepsilon}\left(T^{k}+T^{2 k}\right)$ for $k \geq 4$.

Thus combining (33) and (35) with (28), we obtain the desired inequality (17). This concludes the proof of Proposition 2.

\section{Controllability problems}

\subsection{Observability inequalities for the adjoint system}

Proposition 3. There exists a constant $C_{1}>0$ depending on $\|\hat{u}\|_{\hat{Z}},\|\hat{b}\|_{W^{1, \infty}(0, T)}$, $\|\hat{r}\|_{L^{\infty}(0, T)}$ such that for any $\left(\varphi_{T}, a_{0}^{T}, a_{1}^{T}, \omega_{T}\right)$ with $\varphi_{T} \in L^{2}\left(\widehat{\Omega}_{F}(T)\right)$ and any $(\hat{u}, \hat{b}, \hat{r})$ satisfying (10)-(12), the solution $(\varphi, \pi, a, \omega)$ of (15) satisfies

$$
\|\varphi(0, \cdot)\|_{L^{2}\left(\Omega_{F}(0)\right)}^{2}+|\dot{a}(0)|^{2}+|\omega(0)|^{2} \leq C_{1} \iint_{(0, T) \times \mathcal{O}_{2}}|\varphi|^{2} d x d t .
$$

Proof. The proof relies on an energy inequality for system (15). Indeed, let us multiply the equation for $\varphi$ by $\varphi$ and integrate in space. Using the equations for $a$ and $\omega$, this yields

$$
-\frac{1}{2} \frac{d}{d t} \int_{\widehat{\Omega}_{F}(t)}|\varphi|^{2} d x+\int_{\widehat{\Omega}_{F}(t)}|\nabla \varphi|^{2} d x-\frac{m}{2} \frac{d}{d t}|\dot{a}|^{2}-\frac{1}{2} \frac{d}{d t}(\hat{J} \omega \cdot \omega)=\frac{1}{2} \dot{\hat{J}} \omega \cdot \omega .
$$

Thus, for any $0 \leq t_{1}<t_{2} \leq T$, we have

$\int_{\widehat{\Omega}_{F}\left(t_{1}\right)}\left|\varphi\left(t_{1}\right)\right|^{2} d x+\left|\dot{a}\left(t_{1}\right)\right|^{2}+\left|\omega\left(t_{1}\right)\right|^{2} \leq \widehat{C}\left(\int_{\widehat{\Omega}_{F}\left(t_{2}\right)}\left|\varphi\left(t_{2}\right)\right|^{2} d x+\left|\dot{a}\left(t_{2}\right)\right|^{2}+\left|\omega\left(t_{2}\right)\right|^{2}\right)$.

Combining this with the Carleman inequality (17) and using the properties of the weight function $\alpha$ (see (16)), we obtain (36) in a classical way.

The observability inequality (36) will not allow us to drive the center of mass $a$ to zero at time $t=T$ and the rotation matrix $Q$ to the identity at time $t=T$. For this, we will improve this observability inequality (see (39) below), following the ideas of [15]. 
We first introduce some auxiliary problems. Let us denote by $e_{k}$ the $k$-th element of the canonical basis in $\mathbb{R}^{3}$ for $k=1,2,3$. Let $\left(\varphi^{(j)}, \pi^{(j)}, a^{(j)}, \omega^{(j)}\right)$ be the solution of

$$
\begin{cases}\left.-\varphi_{t}^{(j)}(t, x)-(\hat{u} \cdot \nabla) \varphi^{(j)}\right)(t, x)-\nabla \cdot \sigma\left(\varphi^{(j)}, \pi^{(j)}\right)(t, x)=0, & x \in \widehat{\Omega}_{F}(t), \\ \nabla \cdot \varphi^{(j)}(t, x)=0, & x \in \widehat{\Omega}_{F}(t), \\ \varphi^{(j)}(t, x)=0, & x \in \partial \Omega, \\ \varphi^{(j)}(t, x)=\dot{a}^{(j)}(t)+\omega^{(j)}(t) \times(x-\hat{b}(t)), & x \in \partial \widehat{\Omega}_{S}(t), \\ m\left(\ddot{a}^{(j)}(t)+e_{j}\right)=-\int_{\partial \widehat{\Omega}_{S}(t)}\left(\sigma\left(\varphi^{(j)}, \pi^{(j)}\right) n\right)(t, x) d \gamma, & \\ \frac{d}{d t}\left(\hat{J} \omega^{(j)}\right)(t)=\left((\hat{J} \hat{r}) \times \omega^{(j)}\right)(t)-\int_{\partial \widehat{\Omega}_{S}(t)}(x-\hat{b}(t)) \times\left(\sigma\left(\varphi^{(j)}, \pi^{(j)}\right) n\right)(t, x) d \gamma \\ \varphi_{\mid t=T}^{(j)}=0 \quad \text { in } \widehat{\Omega}_{F}(T), \quad a^{(j)}(T)=\dot{a}^{(j)}(T)=\omega^{(j)}(T)=0,\end{cases}
$$

for $j=1,2,3$ and the solution of

$$
\begin{cases}\left.-\varphi_{t}^{(j)}(t, x)-(\hat{u} \cdot \nabla) \varphi^{(j)}\right)(t, x)-\nabla \cdot \sigma\left(\varphi^{(j)}, \pi^{(j)}\right)(t, x)=0, & x \in \widehat{\Omega}_{F}(t), \\ \nabla \cdot \varphi^{(j)}(t, x)=0, & x \in \widehat{\Omega}_{F}(t), \\ \varphi^{(j)}(t, x)=0, & x \in \partial \Omega, \\ \varphi^{(j)}(t, x)=\dot{a}^{(j)}(t)+\omega^{(j)}(t) \times(x-\hat{b}(t)), & x \in \partial \widehat{\Omega}_{S}(t), \\ m \ddot{a}^{(j)}(t)=-\int_{\partial \widehat{\Omega}_{S}(t)}\left(\sigma\left(\varphi^{(j)}, \pi^{(j)}\right) n\right)(t, x) d \gamma, & \\ \frac{d}{d t}\left(\hat{J} \omega^{(j)}\right)(t)+e_{j-3}=\left((\hat{J} \hat{r}) \times \omega^{(j)}\right)(t)-\int_{\partial \widehat{\Omega}_{S}(t)}(x-\hat{b}(t)) \times\left(\sigma\left(\varphi^{(j)}, \pi^{(j)}\right) n\right)(t, x) d \gamma, \\ \varphi_{\mid t=T}^{(j)}=0 \quad \text { in } \widehat{\Omega}_{F}(T), \quad a^{(j)}(T)=\dot{a}^{(j)}(T)=\omega^{(j)}(T)=0,\end{cases}
$$

for $j=4,5,6$.

Using the duality between systems (37)-(38) and (14), we obtain

$$
\begin{aligned}
\int_{0}^{T} \int_{\mathcal{O}_{2}} v^{*} \cdot \varphi^{(j)} d x d t= & -\int_{\Omega_{F}(0)} u_{0} \cdot \varphi_{\mid t=0}^{(j)} d x-m \dot{a}^{(j)}(0) \cdot b_{1}-\hat{J}(0) r_{0} \cdot \omega^{(j)}(0) \\
& +m\left(b_{j}^{*}(T)-b_{0, j}\right)
\end{aligned}
$$

for $j=1,2,3$ and

$$
\begin{aligned}
\int_{0}^{T} \int_{\mathcal{O}_{2}} v^{*} \cdot \varphi^{(j)} d x d t= & -\int_{\Omega_{F}(0)} u_{0} \cdot \varphi_{\mid t=0}^{(j)} d x-m \dot{a}^{(j)}(0) \cdot b_{1}-\hat{J}(0) r_{0} \cdot \omega^{(j)}(0) \\
& +\int_{0}^{T} r_{j-3}^{*}(t) d t
\end{aligned}
$$

for $j=4,5,6$. 
Observe that $b_{j}^{*}(T)=0$ for $j=1,2,3$ is equivalent to $v^{*}$ satisfying three conditions depending on $u_{0}, b_{0}, b_{1}$ and $r_{0}$. On the other hand, if we denote by $\theta_{0}$ and $\left(x_{0}, x_{1}, x_{2}\right)$ respectively the angle and the axis of the rotation matrix $Q_{0}$, we have

$$
Q_{0}=\exp \left[\left(\begin{array}{ccc}
0 & -x_{2} \theta_{0} & x_{1} \theta_{0} \\
x_{2} \theta_{0} & 0 & -x_{0} \theta_{0} \\
-x_{1} \theta_{0} & x_{0} \theta_{0} & 0
\end{array}\right)\right]
$$

Note also that from (3) we get

$$
Q^{*}(t)=\exp \left[\int_{0}^{t}\left(\begin{array}{ccc}
0 & -r_{3}^{*} & r_{2}^{*} \\
r_{3}^{*} & 0 & -r_{1}^{*} \\
-r_{2}^{*} & r_{1}^{*} & 0
\end{array}\right)(\tau) d \tau\right] Q_{0} .
$$

Thus, $Q^{*}(T)=$ Id will hold if

$$
\int_{0}^{T} r_{3}^{*}(t) d t=-x_{2} \theta_{0}, \quad \int_{0}^{T} r_{2}^{*}(t) d t=-x_{1} \theta_{0}, \quad \int_{0}^{T} r_{1}^{*}(t) d t=-x_{0} \theta_{0},
$$

which is equivalent to three conditions on the control $v^{*}$ depending on $u_{0}, b_{1}, r_{0}$ and $Q_{0}$.

As a conclusion, enforcing $b^{*}(T)=0$ and $Q^{*}(T)=\mathrm{Id}$ is equivalent to

$$
\int_{0}^{T} \int_{\mathcal{O}_{2}} v^{*}(t, x) \cdot \varphi^{(j)}(t, x) d x d t=C^{(j)} \quad \forall 1 \leq j \leq 6,
$$

for some $C^{(j)} \in \mathbb{R}$ depending on the initial conditions. Observe that the set of functions $v^{*}$ satisfying this system of equations is nonempty. Indeed, assume that a linear combination of $\left\{\varphi^{(j)}\right\}_{1 \leq j \leq 6}$ vanishes on $\mathcal{O}_{2}$; then according to the unique continuation property of the fluid problem proved in [8], it vanishes on the whole fluid domain. Then due to the solid equations, we can show that the coefficients of the linear combination are null (we refer to [4] for more details).

We define the orthogonal projection $P$ from $L^{2}((0, T) \times \Omega)$ to $\operatorname{span}\left(1_{\mathcal{O}_{2}}\left(\varphi^{(j)}\right)_{1 \leq j \leq 6}\right)$ :

$$
\int_{0}^{T} \int_{\mathcal{O}_{2}}\left(v^{*}-P\left(v^{*}\right)\right) \cdot \varphi^{(j)} d x d t=0, \quad 1 \leq j \leq 6 .
$$

We also consider the operators $P^{(j)}$ satisfying

$$
P\left(v^{*}\right)=\sum_{j=1}^{6} P^{(j)}\left(v^{*}\right) \varphi^{(j)}
$$

Proposition 4. There exists $C_{1}>0$ depending on $\|\hat{u}\|_{\hat{Z}},\|\hat{b}\|_{W^{1, \infty}(0, T)},\|\hat{r}\|_{L^{\infty}(0, T)}$ such that for any $\left(\varphi_{T}, a_{0}^{T}, a_{1}^{T}, \omega_{T}\right)$ with $\varphi_{T} \in L^{2}\left(\widehat{\Omega}_{F}(T)\right)$ and any $(\hat{u}, \hat{b}, \hat{r})$ satisfying (10)(12), the solution $(\varphi, \pi, a, \omega)$ of (15) satisfies

$$
\begin{aligned}
\|\varphi(0, \cdot)\|_{L^{2}\left(\Omega_{F}(0)\right)}^{2}+|\dot{a}(0)|^{2}+|\omega(0)|^{2} & +\sum_{j=1}^{6}\left|P^{(j)}(\varphi)\right|^{2} \\
& \leq C_{1} \iint_{(0, T) \times \mathcal{O}_{2}}|\varphi-P(\varphi)|^{2} d x d t .
\end{aligned}
$$


The idea of the proof is to argue by contradiction and use the Carleman inequality (17). This is done in the same way as in [7, Proposition 3.2] and [4, Proposition 5], so we omit the proof.

\subsection{Controllability of system (13)}

In this subsection, we prove the null controllability of system (13):

Proposition 5. Let $\left(u_{0}, b_{0}, b_{1}, r_{0}\right)$ satisfy (8), $u_{0} \in H^{2}\left(\Omega_{F}(0)\right)$ and $(\hat{u}, \hat{b}, \hat{r})$ satisfy (10)-(12). Then there exists a control $v \in L^{2}\left(0, T ; H^{1}(\Omega)\right)$ such that the solution $(u, p, b, r)$ to the problem (13) satisfies

$$
u(T, \cdot)=0 \text { in } \widehat{\Omega}_{F}(T), \quad b(T)=0, \dot{b}(T)=0, \omega(T)=0, Q(T)=\mathrm{Id},
$$

where $Q$ is given by (3). Moreover, there exists a constant $K_{0}>0$ such that

$$
\|v\|_{L^{2}\left(0, T ; H^{1}(\Omega)\right)} \leq K_{0}\left(\left\|u_{0}\right\|_{H^{2}\left(\Omega_{F}(0)\right)}+\left|b_{0}\right|+\left|b_{1}\right|+\left|r_{0}\right|\right) .
$$

Proof. From the observability inequality (39), it is classical to prove the existence of a control $v^{*} \in L^{2}((0, T) \times \Omega)$ such that the solution

$$
\left(u^{*}, p^{*}, b^{*}, r^{*}\right) \in\left(L^{2}\left(H^{2}\right) \cap C^{0}\left(H^{1}\right)\right) \times L^{2}\left(H^{1}\right) \times H^{2}(0, T) \times H^{1}(0, T)
$$

of (14) satisfies (40) and (41) for the $L^{2}\left(L^{2}\right)$ norm (see [7, Proposition 4.1] [4, Proposition 6]). In the rest of this proof, $\widehat{C}$ denotes a generic positive constant which may depend on $\|\hat{u}\|_{\hat{Z}},\|\hat{b}\|_{W^{1, \infty}(0, T)}$ and $\|\hat{r}\|_{L^{\infty}(0, T)}$.

Let us now modify the control $v^{*}$ to an $L^{2}\left(H^{1}\right)$ control such that (40) and (41) are still satisfied. For this purpose, let $(\bar{u}, \bar{p}, \bar{b}, \bar{r})$ be the solution of (13) with null control. From Corollary 9 for $k_{0}=1$, we have

$$
(\bar{u}, \bar{p}, \bar{b}, \bar{r}) \in\left(L^{2}\left(H^{3}\right) \cap C^{0}\left(H^{2}\right)\right) \times L^{2}\left(H^{2}\right) \times H^{5 / 2}(0, T) \times H^{3 / 2}(0, T)
$$

and there exists $K>0$ such that

$$
\begin{aligned}
\|\bar{u}\|_{L^{2}\left(H^{3}\right)}+\|\bar{u}\|_{C^{0}\left(H^{2}\right)}+\|\bar{p}\|_{L^{2}\left(H^{2}\right)} & +\|\bar{b}\|_{H^{5 / 2}(0, T)}+\|\bar{r}\|_{H^{3 / 2}(0, T)} \\
\leq & \widehat{C}\left(\left\|u_{0}\right\|_{H^{2}\left(\Omega_{F}(0)\right)}+\left|b_{0}\right|+\left|b_{1}\right|+\left|r_{0}\right|\right) .
\end{aligned}
$$

We now consider a function $\eta_{0} \in C^{1}([0, T])$ such that $\eta_{0}(t)=1, t \in[0, T / 2]$, $\eta_{0}(t)=0, t \in[3 T / 4, T]$ and $\eta_{0}(t) \geq 0, t \in[0, T]$. Then the function

$$
(w, q, c, s):=\left(u^{*}-\eta_{0} \bar{u}, p^{*}-\eta_{0} \bar{p}, b^{*}-\eta_{0} \bar{b}, r^{*}-\eta_{0} \bar{r}\right)
$$


satisfies the first four identities of (40) and the system

$$
\begin{cases}\left.w_{t}(t, x)+(\hat{u} \cdot \nabla) w\right)(t, x)-\nabla \cdot \sigma(w, q)(t, x)=F_{0}(t, x)+v^{*} 1_{\mathcal{O}_{2},}, & x \in \widehat{\Omega}_{F}(t), \\ \nabla \cdot w(t, x)=0, & x \in \widehat{\Omega}_{F}(t), \\ w(t, x)=0, & x \in \partial \Omega, \\ w(t, x)=\dot{c}(t)+s(t) \times(x-\hat{b}(t))+F_{1}(t), & x \in \partial \widehat{\Omega}_{S}(t), \\ m \ddot{c}(t)=\int_{\partial \widehat{\Omega}_{S}(t)}(\sigma(w, q) n)(t, x) d \gamma+F_{2}(t), & \\ (\hat{J} \dot{S})(t)=((\hat{J} \hat{r}) \times s)(t)+\int_{\partial \widehat{\Omega}_{S}(t)}(x-\hat{b}(t)) \times(\sigma(w, q) n)(t, x) d \gamma+F_{3}(t), \\ w_{\mid t=0}=0 \quad \text { in } \Omega_{F}(0), \quad c(0)=0, \dot{c}(0)=0, s(0)=0,\end{cases}
$$

where $F_{0}:=-\eta_{0, t} \bar{u} \in L^{2}\left(H^{2}\right), F_{1}=\eta_{0, t} \bar{b}, F_{2}:=-m\left(\eta_{0, t t} \bar{b}+2 \eta_{0, t} \dot{\bar{b}}\right) \in H^{1}(0, T)$ and $F_{3}:=-\eta_{0, t} \hat{J} \bar{r} \in H^{1}(0, T)$. Thanks to (42), we have

$$
\begin{aligned}
\left\|F_{0}\right\|_{L^{2}\left(H^{2}\right)}+\left\|F_{1}\right\|_{H^{1}(0, T)}+\left\|F_{2}\right\|_{H^{1}(0, T)}+\left\|F_{3}\right\|_{H^{1}(0, T)} & \\
& \leq \widehat{C}\left(\left\|u_{0}\right\|_{H^{2}\left(\Omega_{F}(0)\right)}+\left|b_{0}\right|+\left|b_{1}\right|+\left|r_{0}\right|\right) .
\end{aligned}
$$

Using this estimate and Proposition 7, we obtain

$$
\begin{aligned}
\|w\|_{L^{2}\left(H^{2}\right)}+\|w\|_{H^{1}\left(L^{2}\right)}+\|q\|_{L^{2}\left(H^{1}\right)} & +\|c\|_{H^{2}(0, T)}+\|s\|_{H^{1}(0, T)} \\
\leq & \widehat{C}\left(\left\|u_{0}\right\|_{H^{2}\left(\Omega_{F}(0)\right)}+\left|b_{0}\right|+\left|b_{1}\right|+\left|r_{0}\right|\right) .
\end{aligned}
$$

We consider two open sets $\mathcal{O}_{3}$ and $\mathcal{O}_{4}$ such that

$$
\mathcal{O}_{2} \subset \subset \mathcal{O}_{3} \subset \subset \mathcal{O}_{4} \subset \subset \widetilde{\mathcal{O}} \text {. }
$$

Let $\theta \in C_{c}^{2}\left(\mathcal{O}_{4}\right)$ be a function satisfying $\theta(x)=1$ for every $x \in \mathcal{O}_{3}$. We introduce the functions

$$
(\tilde{w}, \tilde{q}, \tilde{c}, \tilde{s}):=((1-\theta) w,(1-\theta) q, c, s),
$$

which satisfy the first four identities of (40) and the following system:

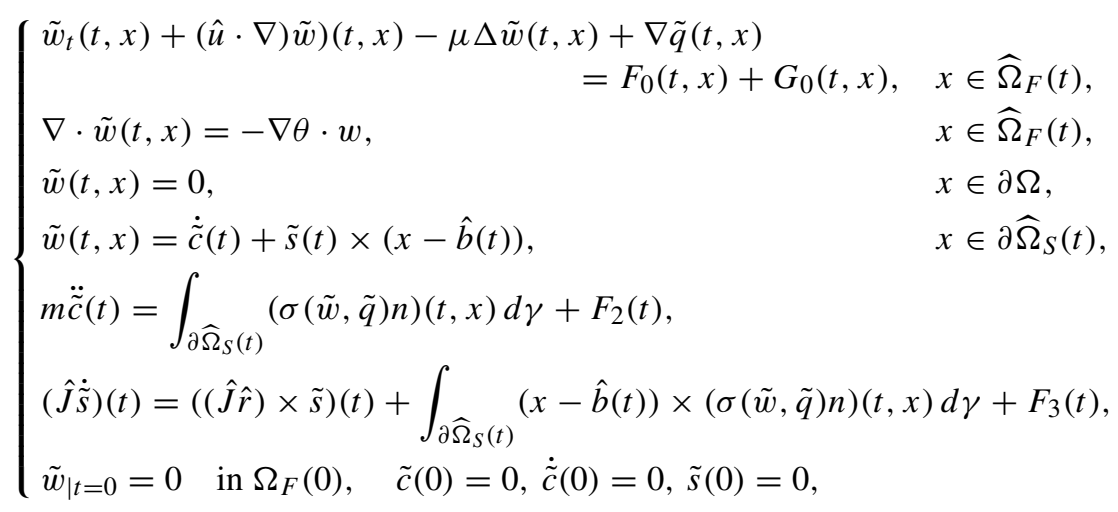


with

$$
G_{0}:=-\theta F_{0}-(\hat{u} \cdot \nabla \theta) w+\mu(2(\nabla \theta \cdot \nabla) w+\Delta \theta w)-q \nabla \theta .
$$

Here, we have used that $(1-\theta) v^{*} 1_{\mathcal{O}_{2}} \equiv 0$. Using (12), the properties of $\theta,(44)$ and (45), we have

$$
\operatorname{supp}\left(G_{0}\right) \subset \mathcal{O}_{4}, \quad\left\|G_{0}\right\|_{L^{2}\left(H^{1}\right)} \leq \widehat{C}\left(\left\|u_{0}\right\|_{H^{2}\left(\Omega_{F}(0)\right)}+\left|b_{0}\right|+\left|b_{1}\right|+\left|r_{0}\right|\right) .
$$

Let us now lift the divergence term. This term satisfies

$$
\operatorname{supp}(\nabla \theta \cdot w) \subset \subset \mathcal{O}_{4}, \quad \int_{\mathcal{O}_{4}} \nabla \theta \cdot w d x=0, \quad \nabla \theta \cdot w \in L^{2}\left(H^{2}\right) \cap H^{1}\left(L^{2}\right)
$$

By [2, Theorem 2.4, p. 72 with $m=r=2]$, there exists a lifting $U \in H^{1}\left(H_{0}^{1}\left(\mathcal{O}_{4}\right)\right)$ $\cap L^{2}\left(H_{0}^{3}\left(\mathcal{O}_{4}\right)\right)$ satisfying

$$
\nabla \cdot U=\nabla \theta \cdot w \text { in } \mathcal{O}_{4},\|U\|_{H^{1}\left(H^{1}\right)}+\|U\|_{L^{2}\left(H^{3}\right)} \leq \widehat{C}\left(\|w\|_{H^{1}\left(L^{2}\right)}+\|w\|_{L^{2}\left(H^{2}\right)}\right)
$$

Moreover, since $w_{\mid t=0}=w_{t=T}=0$ in $\mathcal{O}_{4}$, we have $U_{\mid t=0}=U_{\mid t=T}=0$ in $\mathcal{O}_{4}$. Let us still denote by $U$ its extension by zero to $\Omega$. We now consider the system satisfied by $(W:=\tilde{w}-U, \tilde{q}, \tilde{c}, \tilde{s}):$

$$
\begin{cases}\left.W_{t}(t, x)+(\hat{u} \cdot \nabla) W\right)(t, x)-\nabla \cdot \sigma(W, \tilde{q})(t, x)=F_{0}(t, x)+G_{1}(t, x), & x \in \widehat{\Omega}_{F}(t), \\ \nabla \cdot W(t, x)=0, & x \in \widehat{\Omega}_{F}(t), \\ W(t, x)=0, & x \in \partial \Omega, \\ W(t, x)=\dot{\tilde{c}}(t)+\tilde{s}(t) \times(x-\hat{b}(t)), & x \in \partial \widehat{\Omega}_{S}(t), \\ m \ddot{\tilde{c}}(t)=\int_{\partial \widehat{\Omega}_{S}(t)}(\sigma(W, \tilde{q}) n)(t, x) d \gamma+F_{2}(t), & \\ (\hat{J} \dot{\tilde{s}})(t)=((\hat{J} \hat{r}) \times \tilde{s})(t)+\int_{\partial \widehat{\Omega}_{S}(t)}(x-\hat{b}(t)) \times(\sigma(W, \tilde{q}) n)(t, x) d \gamma+F_{3}(t), & \\ W_{\mid t=0}=0 \quad \text { in } \Omega_{F}(0), \quad \tilde{c}(0)=0, \dot{\tilde{c}}(0)=0, \tilde{s}(0)=0, & \end{cases}
$$

with

$$
G_{1}:=G_{0}-U_{t}-(\hat{u} \cdot \nabla) U+\mu \Delta U .
$$

From the definition of $\theta,(47),(48)$ and the fact that $\hat{u} \in \widehat{Z}$, it is clear that

$$
\operatorname{supp}\left(G_{1}\right) \subset \mathcal{O}_{4}, \quad\left\|G_{1}\right\|_{L^{2}\left(H^{1}\right)} \leq \widehat{C}\left(\left\|u_{0}\right\|_{H^{2}\left(\Omega_{F}(0)\right)}+\left|b_{0}\right|+\left|b_{1}\right|+\left|r_{0}\right|\right) .
$$

Consequently, we have $G_{1}=\zeta G_{1}$ and $v:=G_{1}$ satisfies (41). Finally, $(u, p, b, r):=$ $\left(W+\eta_{0} \bar{u}, \tilde{q}+\eta_{0} \bar{p}, \tilde{c}+\eta_{0} \bar{b}, \tilde{s}+\eta_{0} \bar{r}\right)$ with the control force $v$ solves system (13), and since $r=r^{*}$ and $Q(T)=Q^{*}(T)=\mathrm{Id}$, (40) holds. 


\section{Local null controllability}

To prove Theorem 1, we perform a fixed-point argument for a multivalued map (see [20, Theorem 9.B, p. 452]):

Theorem 6. Assume that a multivalued map $\Lambda: K \rightarrow 2^{K}$ satisfies:

- $\Lambda$ is upper semicontinuous.

- $K$ is a nonempty, compact, convex set in a locally convex space $X$.

- The set $\Lambda(x)$ is nonempty, closed and convex for all $x \in K$.

Then $\Lambda$ has a fixed point.

We are going to apply this theorem in the fixed domain $\Omega_{F}(0)$. More precisely, let

$K:=\left\{(z, b, r) \in\left(L^{2}\left(0, T ; W^{2,6}\left(\Omega_{F}(0)\right)\right) \cap H^{1}\left(0, T ; L^{6}\left(\Omega_{F}(0)\right)\right)\right) \times H^{2}(0, T) \times H^{1}(0, T)\right.$

such that $\nabla \cdot z=0$ in $\Omega_{F}(0), z=0$ on $\partial \Omega_{F}(0)$ and

$\left.\|z\|_{L^{2}\left(0, T ; W^{2,6}\left(\Omega_{F}(0)\right)\right)}+\|z\|_{H^{1}\left(0, T ; L^{6}\left(\Omega_{F}(0)\right)\right)}+\|b\|_{H^{2}(0, T)}+\|r\|_{H^{1}(0, T)} \leq R\right\}$

for some small $R>0$, and

$$
X:=L^{2}\left(0, T ; H^{1}\left(\Omega_{F}(0)\right)\right) \times C^{1}([0, T]) \times C^{0}([0, T]) .
$$

In order to define $\Lambda$, we consider $(\hat{z}, \hat{b}, \hat{r}) \in K$. We define the associated flow in the solid domain:

$$
\hat{\chi}(t, y)=\hat{b}(t)+\hat{Q}(t) Q_{0}^{-1}\left(y-b_{0}\right) \quad \forall y \in \Omega_{S}(0) .
$$

Then the solid domain is given by $\widehat{\Omega}_{S}(t):=\hat{\chi}\left(t, \Omega_{S}(0)\right)$ for each $t>0$. Observe that condition (11) is satisfied for $R$ small enough. Next, we define the eulerian velocity $\hat{u}_{S} \in$ $H^{1}\left(H^{3}\right)$ as the solution, together with $\hat{q}_{S}$, of

$$
\begin{cases}-\mu \Delta \hat{u}_{S}+\nabla \hat{q}_{S}=0 & \text { in } \widehat{\Omega}_{F}(t), \\ \nabla \cdot \hat{u}_{S}=0 & \text { in } \widehat{\Omega}_{F}(t), \\ \hat{u}_{S}(t, x)=\dot{\hat{b}}(t)+\hat{r}(t) \times(x-\hat{b}(t)) & \text { on } \partial \widehat{\Omega}_{S}(t), \\ \hat{u}_{S}=0 & \text { on } \partial \Omega\end{cases}
$$

It satisfies

$$
\left\|\hat{u}_{S}\right\|_{H^{1}\left(H^{3}\right)}+\left\|\hat{q}_{S}\right\|_{H^{1}\left(H^{2}\right)} \leq C\left(\|\hat{b}\|_{H^{2}(0, T)}+\|\hat{r}\|_{H^{1}(0, T)}\right)
$$

for some $C>0$.

Now, we extend the flow $\hat{\chi}$ to the fluid domain:

$$
\left\{\begin{array}{l}
\frac{\partial \hat{\chi}(t, y)}{\partial t}=\left(\hat{u}_{S} \circ \hat{\chi}\right)(t, y) \quad \forall y \in \Omega_{F}(0), \\
\hat{\chi}(0, y)=y \quad \forall y \in \Omega_{F}(0) .
\end{array}\right.
$$


This flow satisfies

$$
\|\hat{\chi}-\mathrm{id}\|_{H^{2}\left(H^{3}\right)} \leq C\left(\|\hat{b}\|_{H^{2}(0, T)}+\|\hat{r}\|_{H^{1}(0, T)}\right) \leq C R
$$

for some $C>0$.

Next, we consider $\hat{u} \in \hat{Z}$ defined by

$$
\hat{u}(t, x):=\hat{u}_{S}(t, x)+\left(\nabla \hat{\chi}\left(t, \hat{\chi}^{-1}(t, x)\right)\right) \hat{z}\left(t, \hat{\chi}^{-1}(t, x)\right) \quad \forall x \in \widehat{\Omega}_{F}(t) .
$$

This vector field satisfies $\nabla \cdot \hat{u}=0$ in $\widehat{\Omega}_{F}(t), \hat{u}=0$ on $\partial \Omega$. Moreover, there exists $C>0$ such that

$$
\|\hat{u}\|_{\hat{Z}} \leq C\left(\|\hat{z}\|_{L^{2}\left(0, T ; W^{2,6}\left(\Omega_{F}(0)\right)\right) \cap H^{1}\left(0, T ; L^{6}\left(\Omega_{F}(0)\right)\right)}+\|\hat{b}\|_{H^{2}(0, T)}+\|\hat{r}\|_{H^{1}(0, T)}\right) .
$$

This velocity vector field being given, according to Proposition 5 we can construct a control $v \in L^{2}\left(0, T ; H^{1}(\Omega)\right)$ and a solution $(u, p, b, r)$ of system (13) which satisfy (40) and (41). From Proposition 7 (with $g_{0}=g_{2}=g_{3}=0$ and $g_{1}=v \zeta(x)$ ), we infer $(u, p, b, r) \in \hat{Y}_{0} \times L^{2}\left(H^{1}\right) \times H^{2}(0, T) \times H^{1}(0, T)$ and

$$
\begin{aligned}
& \|(u, p, b, r)\|_{\hat{Y}_{0} \times L^{2}\left(H^{1}\right) \times H^{2}(0, T) \times H^{1}(0, T)} \\
& \quad \leq \widehat{C}\left(\|v\|_{L^{2}((0, T) \times \Omega)}+\left\|u_{0}\right\|_{H^{1}\left(\Omega_{F}(0)\right)}+\left|b_{0}\right|+\left|b_{1}\right|+\left|r_{0}\right|\right) .
\end{aligned}
$$

Let $\left(u_{S}, q_{S}\right)$ be defined by (52) with the boundary condition on $\partial \widehat{\Omega}_{S}(t)$ replaced by $\dot{b}(t)+r(t) \times(x-\hat{b}(t))$. Then $\left(u_{S}, q_{S}\right)$ satisfies $(53)$ with $(\hat{b}, \hat{r})$ replaced by $(b, r)$ and $\left(u-u_{S}, p-q_{S}\right)$ is the solution of the following system:

$$
\begin{cases}\left(u-u_{S}\right)_{t}(t, x)-\nabla \cdot \sigma\left(u-u_{S}, p-q_{S}\right)(t, x) & \\ \quad=v \zeta(x)-(\hat{u} \cdot \nabla) u(t, x)-u_{S, t}(t, x), & x \in \widehat{\Omega}_{F}(t), \\ \nabla \cdot\left(u-u_{S}\right)(t, x)=0, & x \in \widehat{\Omega}_{F}(t), \\ \left(u-u_{S}\right)(t, x)=0, & x \in \partial \widehat{\Omega}_{F}(t), \\ \left(u-u_{S}\right)(0, x)=u_{0}(x)-u_{S}(0, x), & x \in \Omega_{F}(0) .\end{cases}
$$

Since $v \in L^{2}\left(0, T ; H^{1}(\Omega)\right), \hat{u} \in \hat{Z}, u \in \hat{Y}_{0}$ and $u_{S}$ satisfies (53), the right-hand side of this system belongs to $L^{2}\left(L^{6}\right)$.

Finally, we define

$$
z(t, y):=(\nabla \hat{\chi})^{-1}(t, y)\left(u-u_{S}\right)(t, \hat{\chi}(t, y)) \quad \forall y \in \Omega_{F}(0)
$$

and $h(t, y):=\left(p-q_{S}\right)(t, \hat{\chi}(t, y))$ for $y \in \Omega_{F}(0)$. We notice that $(z, h)$ satisfies

$$
\begin{cases}z_{t}-\nabla \cdot \sigma(z, h)=F & \text { in }(0, T) \times \Omega_{F}(0), \\ \nabla \cdot z=0 & \text { in }(0, T) \times \Omega_{F}(0), \\ z=0 & \text { on }(0, T) \times \partial \Omega_{F}(0), \\ z(0, x)=u_{0}(x)-u_{S}(0, x) & \text { in } \Omega_{F}(0),\end{cases}
$$


where

$$
\begin{aligned}
\|F\|_{L^{2}\left(0, T ; L^{6}\left(\Omega_{F}(0)\right)\right)} & \\
\leq & C\left(\|v\|_{L^{2}\left(0, T ; H^{1}(\Omega)\right)}+\|\nabla \hat{\chi}-\mathrm{Id}\|_{C^{0}\left([0, T] \times \overline{\left.\Omega_{F}(0)\right)}\right.}\left(\|z\|_{K_{1}}+\|\nabla h\|_{L^{2}\left(0, T ; L^{6}\left(\Omega_{F}(0)\right)\right)}\right)\right) \\
& +\widehat{C}\left(\|u\|_{\hat{Y}_{0}}+\|p\|_{L^{2}\left(H^{1}\right)}+\|b\|_{H^{2}(0, T)}+\|r\|_{H^{1}(0, T)}\right) .
\end{aligned}
$$

Here, $K_{1}$ stands for the first component of the space $K$, which was defined in (50). Now, we decompose $F=F_{1}+\nabla F_{2}$ with $F_{1} \in L^{2}\left(0, T ; L^{6}\left(\Omega_{F}(0)\right)\right)$ satisfying $\nabla \cdot F_{1}=0$ in $(0, T) \times \Omega_{F}(0), F_{1} \cdot n=0$ on $(0, T) \times \partial \Omega_{F}(0), F_{2} \in L^{2}\left(0, T ; W^{1,6}\left(\Omega_{F}(0)\right)\right)$ and

$$
\left\|F_{1}\right\|_{L^{2}\left(0, T ; L^{6}\left(\Omega_{F}(0)\right)\right)}+\left\|\nabla F_{2}\right\|_{L^{2}\left(0, T ; L^{6}\left(\Omega_{F}(0)\right)\right)} \leq C\|F\|_{L^{2}\left(0, T ; L^{6}\left(\Omega_{F}(0)\right)\right)} .
$$

Then, we apply [11, Theorem 2.8] to $\left(z, h-F_{2}\right)$ with right-hand side $F_{1}$ to obtain

$$
\|(z, \nabla h)\|_{K_{1} \times L^{2}\left(0, T ; L^{6}\left(\Omega_{F}(0)\right)\right)} \leq C\left(\|F\|_{L^{2}\left(0, T ; L^{6}\left(\Omega_{F}(0)\right)\right)}+\left\|u_{0}-u_{S}(0, \cdot)\right\|_{H^{2}\left(\Omega_{F}(0)\right)}\right) .
$$

Using now that $(\hat{u}, \hat{b}, \hat{r})$ belongs to $K$, and (55) and (56), we deduce that

$$
\begin{aligned}
\|(z, \nabla h)\|_{K_{1} \times L^{2}\left(0, T ; L^{6}\left(\Omega_{F}(0)\right)\right)} & \leq \widehat{C} R\|(z, \nabla h)\|_{K_{1} \times L^{2}\left(0, T ; L^{6}\left(\Omega_{F}(0)\right)\right)} \\
+ & \widehat{C}\left(\|v\|_{L^{2}\left(0, T ; H^{1}(\Omega)\right)}+\left\|u_{0}\right\|_{H^{2}\left(\Omega_{F}(0)\right)}+\left|b_{0}\right|+\left|b_{1}\right|+\left|r_{0}\right|\right) .
\end{aligned}
$$

Thanks to (41) and (56), we obtain

$$
\|(z, b, r)\|_{K} \leq C\left(\left\|u_{0}\right\|_{H^{2}\left(\Omega_{F}(0)\right)}+\left|b_{0}\right|+\left|b_{1}\right|+\left|r_{0}\right|\right) .
$$

With all these ingredients, we define

$\Lambda(\hat{z}, \hat{b}, \hat{r})=\{(z, b, r) \in K:(u, p, b, r)$ satisfies (13) for some $p$ and $v,(40)$ and (41) $\}$.

- We directly see that $\Lambda: K \rightarrow 2^{K}$ from (59) and taking $\delta$ in (9) sufficiently small.

- Let us now prove that $\Lambda$ is upper semicontinuous. For this, let $A \subset K$ be a closed subset. We have to prove that $\Lambda^{-1}(A)$ is also closed.

Let $\left(\hat{z}_{n}, \hat{b}_{n}, \hat{r}_{n}\right) \subset \Lambda^{-1}(A)$ be such that $\left(\hat{z}_{n}, \hat{b}_{n}, \hat{r}_{n}\right) \rightarrow(\hat{z}, \hat{b}, \hat{r})$ in $X$. We intend to prove that $(\hat{z}, \hat{b}, \hat{r}) \in \Lambda^{-1}(A)$, that is, there exists $(z, b, r) \in A$ such that $(z, b, r) \in$ $\Lambda(\hat{z}, \hat{b}, \hat{r})$. Let $\left(z_{n}, b_{n}, r_{n}\right) \in \Lambda\left(\hat{z}_{n}, \hat{b}_{n}, \hat{r}_{n}\right) \subset A$. From the definition of $K$, there exists a subsequence $\left(z_{\psi(n)}, b_{\psi(n)}, r_{\psi(n)}\right)$ such that

$\left(z_{\psi(n)}, b_{\psi(n)}, r_{\psi(n)}\right) \rightarrow(z, b, r)$ in $K \quad$ and $\quad\left(z_{\psi(n)}, b_{\psi(n)}, r_{\psi(n)}\right) \rightarrow(z, b, r)$ in $X$.

Since $A$ is closed, we see $(z, b, r) \in A$. It remains to prove that $(z, b, r) \in \Lambda(\hat{z}, \hat{b}, \hat{r})$.

First, we observe that $\hat{\chi}_{\psi(n)} \rightarrow \hat{b}+\hat{Q} Q_{0}^{-1}\left(y-b_{0}\right)$ in $C^{1}\left([0, T] ; H^{3}\left(\Omega_{S}(0)\right)\right)$ (see (51)). Let us prove that

$$
\hat{\chi}_{\psi(n)} \rightarrow \hat{\chi} \quad \text { in } C^{1}\left([0, T] ; H^{3}\left(\Omega_{F}(0)\right)\right) .
$$

For this, we consider the Stokes system satisfied by

$$
\left(\hat{u}_{S, \psi(n)} \circ \hat{\chi}_{\psi(n)}, \hat{q}_{S, \psi(n)} \circ \hat{\chi}_{\psi(n)}\right)-\left(\hat{u}_{S} \circ \hat{\chi}, \hat{q}_{S} \circ \hat{\chi}\right) .
$$


Since $\left(z_{\psi(n)}, b_{\psi(n)}, r_{\psi(n)}\right)$ belongs to $K, \hat{u}_{S, \psi(n)}$ satisfies (53) and $\hat{\chi}_{\psi(n)}$ satisfies (55), one can see that the $H^{1}\left(\Omega_{F}(0)\right)$-norm of the right-hand side and the $H^{2}\left(\Omega_{F}(0)\right)$-norm of the divergence term of this system can be estimated by

$$
\begin{aligned}
C R\left(\left\|\hat{u}_{S, \psi(n)} \circ \hat{\chi}_{\psi(n)}-\hat{u}_{S} \circ \hat{\chi}\right\|_{H^{3}\left(\Omega_{F}(0)\right)}+\left\|\hat{q}_{S, \psi(n)} \circ \hat{\chi}_{\psi(n)}-\hat{q}_{S} \circ \hat{\chi}\right\|_{H^{2}\left(\Omega_{F}(0)\right)}\right. \\
\left.+\left\|\hat{\chi}_{\psi(n)}-\hat{\chi}\right\|_{H^{3}\left(\Omega_{F}(0)\right)}\right) .
\end{aligned}
$$

As far as the boundary term is concerned, we have

$$
\begin{aligned}
& \hat{u}_{S, \psi(n)} \circ \hat{\chi}_{\psi(n)}-\hat{u}_{S} \circ \hat{\chi} \\
& \quad=\dot{\hat{b}}_{\psi(n)}-\dot{\hat{b}}+\left(\hat{r}_{\psi(n)}-\hat{r}\right) \times\left(\hat{Q}\left(y-b_{0}\right)\right)+\hat{r}_{\psi(n)} \times\left(\hat{Q}_{\psi(n)}-\hat{Q}\right)\left(y-b_{0}\right),
\end{aligned}
$$

which tends to zero strongly in $C^{0}\left([0, T] ; H^{5 / 2}\left(\partial \Omega_{S}(0)\right)\right)$. Consequently, thanks to (54), we obtain

$$
\hat{u}_{S, \psi(n)} \circ \hat{\chi}_{\psi(n)}-\hat{u}_{S} \circ \hat{\chi} \rightarrow 0 \quad \text { in } C^{0}\left([0, T] ; H^{3}\left(\Omega_{F}(0)\right)\right)
$$

and (61). Taking a look again at the Stokes system satisfied by (62), we see that the $H^{1}\left(0, T ; H^{1}\left(\Omega_{F}(0)\right)\right)$-norm of the right-hand side and the $H^{1}\left(0, T ; H^{2}\left(\Omega_{F}(0)\right)\right)$-norm of the divergence are estimated by

$$
\begin{array}{r}
C R\left(\left\|\hat{u}_{S, \psi(n)} \circ \hat{\chi}_{\psi(n)}-\hat{u}_{S} \circ \hat{\chi}\right\|_{H^{1}\left(0, T ; H^{3}\left(\Omega_{F}(0)\right)\right)}+\left\|\hat{q}_{S, \psi(n)} \circ \hat{\chi}_{\psi(n)}-\hat{q} \hat{q}_{S} \circ\right\|_{H^{1}\left(0, T ; H^{2}\left(\Omega_{F}(0)\right)\right)}\right. \\
\left.+\left\|\hat{\chi}_{\psi(n)}-\hat{\chi}\right\|_{H^{1}\left(0, T ; H^{3}\left(\Omega_{F}(0)\right)\right)}\right) .
\end{array}
$$

For the boundary term (63), we deduce that its $H^{1}\left(0, T ; H^{5 / 2}\left(\partial \Omega_{S}(0)\right)\right)$-norm is bounded independently of $n$. As a consequence, up to a subsequence, we obtain

$$
\hat{u}_{S, \psi(n)} \circ \hat{\chi}_{\psi(n)}-\hat{u}_{S} \circ \hat{\chi} \rightarrow 0 \quad \text { in } H^{1}\left(0, T ; H^{3}\left(\Omega_{F}(0)\right)\right) .
$$

In the same way, one can prove that

$$
\begin{array}{ll}
u_{S, \psi(n)} \circ \hat{\chi}_{\psi(n)}-u_{S} \circ \hat{\chi} \rightarrow 0 & \text { in } C^{0}\left([0, T] ; H^{3}\left(\Omega_{F}(0)\right)\right), \\
u_{S, \psi(n)} \circ \hat{\chi}_{\psi(n)}-u_{S} \circ \hat{\chi} \rightarrow 0 & \text { in } H^{1}\left(0, T ; H^{3}\left(\Omega_{F}(0)\right)\right) .
\end{array}
$$

We recall the definition of $u_{\psi(n)}$ :

$$
u_{\psi(n)} \circ \hat{\chi}_{\psi(n)}=u_{S, \psi(n)} \circ \hat{\chi}_{\psi(n)}+\left(\nabla \hat{\chi}_{\psi(n)}\right) z_{\psi(n)} \text { in } \Omega_{F}(0) .
$$

Thanks to (60), (61) and (64), one can pass to the limit in the system satisfied by

$$
\left(u_{\psi(n)} \circ \hat{\chi}_{\psi(n)}, p_{\psi(n)} \circ \hat{\chi}_{\psi(n)}, b_{\psi(n)}, r_{\psi(n)}\right)
$$

and deduce that $(u, p, b, r)$ satisfies system (13).

- For each $(\hat{z}, \hat{b}, \hat{r}) \in K, \Lambda(\hat{z}, \hat{b}, \hat{r})$ is closed in $X$. Indeed, let $\left(z_{n}, b_{n}, r_{n}\right) \in \Lambda(\hat{z}, \hat{b}, \hat{r})$ be such that $\left(z_{n}, b_{n}, r_{n}\right) \rightarrow(z, b, r)$ in $X$. Then, arguing as above, one can show that $(z, b, r) \in \Lambda(\hat{z}, \hat{b}, \hat{r})$. In fact, the same convergences can be proved in a simpler way since the domains do not depend on $n$.

Thus, we can apply Theorem 6 to obtain the existence of a fixed point to $\Lambda$. This concludes the proof of Theorem 1 . 


\section{Appendix}

In this Appendix, we will establish some regularity results for a fluid-structure system similar to (13):

$$
\begin{cases}w_{t}(t, x)+(\hat{u} \cdot \nabla) w(t, x)-\nabla \cdot \sigma(w, q)(t, x)=g_{1}(t, x), & x \in \widehat{\Omega}_{F}(t), \\ \nabla \cdot w(t, x)=0, & x \in \widehat{\Omega}_{F}(t), \\ w(t, x)=0, & x \in \partial \Omega, \\ w(t, x)=\dot{c}(t)+s(t) \times(x-\hat{b}(t))+g_{0}(t, x), & x \in \partial \widehat{\Omega}_{S}(t), \\ m \ddot{c}(t)=\int_{\partial \widehat{\Omega}_{S}(t)}(\sigma(w, q) n)(t, x) d \gamma+g_{2}(t), & \\ (\hat{J} \dot{S})(t)=((\hat{J} \hat{r}) \times s)(t)+\int_{\partial \widehat{\Omega}_{S}(t)}(x-\hat{b}(t)) \times(\sigma(w, q) n)(t, x) d \gamma+g_{3}(t), \\ w_{\mid t=0}=w_{0} \quad \text { in } \Omega_{F}(0), \quad c(0)=c_{0}, \dot{c}(0)=c_{1}, s(0)=s_{0} .\end{cases}
$$

Proposition 7. Assume that $\left(w_{0}, c_{0}, c_{1}, s_{0}\right)$ satisfies (8) and let $(\hat{u}, \hat{b}, \hat{r})$ satisfy (10) (with $\left(b_{0}, b_{1}, r_{0}\right)$ replaced by $\left.\left(c_{0}, c_{1}, s_{0}\right)\right)$ and $(11)-(12)$. Moreover, suppose that $g_{0} \in L^{2}\left(H^{2}\right)$, the trace of $g_{0}$ belongs to $H^{1}\left(0, T ; L^{2}\left(\partial \widehat{\Omega}_{S}(t)\right)\right), g_{1} \in L^{2}\left(L^{2}\right), g_{2} \in L^{2}(0, T)$ and $g_{3} \in$ $L^{2}(0, T)$. Then there exists $\widehat{C}$ (depending on $\Omega, \delta_{0}$ and $\|\hat{u}\|_{\hat{Z}},\|\hat{b}\|_{H^{2}(0, T)},\|\hat{r}\|_{H^{1}(0, T)}$ ) such that the solution of (65) satisfies

$$
(w, q, c, s) \in \hat{Y}_{0} \times L^{2}\left(H^{1}\right) \times H^{2}(0, T) \times H^{1}(0, T)
$$

and

$$
\begin{aligned}
\|(w, q, c, s) & \|_{\hat{Y}_{0} \times L^{2}\left(H^{1}\right) \times H^{2}(0, T) \times H^{1}(0, T)} \\
\leq & \widehat{C}\left(\left\|g_{0}\right\|_{L^{2}\left(H^{2}\right)}+\left\|g_{0}\right\|_{H^{1}\left(0, T ; L^{2}\left(\partial \widehat{\Omega}_{S}(t)\right)\right)}+\left\|g_{1}\right\|_{L^{2}\left(L^{2}\right)}+\left\|g_{2}\right\|_{L^{2}(0, T)}\right. \\
& \left.+\left\|g_{3}\right\|_{L^{2}(0, T)}+\left\|w_{0}\right\|_{H^{1}\left(\Omega_{F}(0)\right)}+\left|c_{0}\right|+\left|c_{1}\right|+\left|s_{0}\right|\right) .
\end{aligned}
$$

Proof. First, we prove that $w \in L^{2}\left(H^{1}\right) \cap C^{0}\left(L^{2}\right)$ together with $c \in W^{1, \infty}(0, T), s \in$ $L^{\infty}(0, T)$. Then, we will prove $(w, q, c, s) \in \hat{Y}_{0} \times L^{2}\left(H^{1}\right) \times H^{2}(0, T) \times H^{1}(0, T)$.

First step: We multiply the equation for $w$ in (65) by $w$ and we integrate over $\widehat{\Omega}_{F}(t)$. After an integration by parts and using the equations of the solid, this yields

$$
\begin{aligned}
& \frac{1}{2} \frac{d}{d t} \int_{\widehat{\Omega}_{F}(t)}|w|^{2} d x+\mu \int_{\widehat{\Omega}_{F}(t)}|\nabla w|^{2} d x+\frac{m}{2} \frac{d}{d t}|\dot{c}|^{2}+\frac{1}{2} \frac{d}{d t}\left(\hat{J}_{s} \cdot s\right) \\
& \quad=\int_{\widehat{\Omega}_{F}(t)} w g_{1} d x+\dot{c} \cdot g_{2}+s \cdot g_{3}+\frac{1}{2} \dot{\hat{J}} s \cdot s-\int_{\partial \widehat{\Omega}_{S}(t)}(\sigma(w, q) n) \cdot g_{0} d \gamma
\end{aligned}
$$

We integrate in $t$ and use $\hat{J} s \cdot s \geq C|s|^{2}$ for some $C>0$ to obtain

$$
\begin{aligned}
& \|w\|_{L^{2}\left(H^{1}\right)}+\|w\|_{L^{\infty}\left(L^{2}\right)}+\|c\|_{W^{1, \infty}(0, T)}+\|s\|_{L^{\infty}(0, T)} \\
& \leq \widehat{C}_{\varepsilon}\left(\left\|g_{0}\right\|_{L^{2}\left(H^{2}\right)}+\left\|g_{1}\right\|_{L^{1}\left(L^{2}\right)}+\left\|g_{2}\right\|_{L^{1}(0, T)}+\left\|g_{3}\right\|_{L^{1}(0, T)}+\left\|w_{0}\right\|_{L^{2}\left(\Omega_{F}(0)\right)}\right. \\
& \left.\quad+\left|c_{0}\right|+\left|c_{1}\right|+\left|s_{0}\right|\right)+\varepsilon\|(w, q)\|_{\hat{Y}_{0} \times L^{2}\left(H^{1}\right)}
\end{aligned}
$$

for any $\varepsilon>0$. 
Second step: We multiply the equation for $w$ by $w_{t}$ and we integrate over $\widehat{\Omega}_{F}(t)$. After some computations, we obtain

$$
\begin{aligned}
\int_{\widehat{\Omega}_{F}(t)}\left|w_{t}\right|^{2} d x & +\frac{1}{2} \frac{d}{d t} \int_{\widehat{\Omega}_{F}(t)}|\nabla w|^{2} d x+m|\ddot{c}|^{2}+\hat{J} \dot{s} \cdot \dot{s} \\
= & -\int_{\widehat{\Omega}_{F}(t)} w_{t} \cdot(\hat{u} \cdot \nabla) w d x+\frac{1}{2} \int_{\widehat{\Omega}_{F}(t)} \hat{u} \cdot \nabla|\nabla w|^{2} d x+\int_{\widehat{\Omega}_{F}(t)} w_{t} \cdot g_{1} d x \\
& -\int_{\partial \widehat{\Omega}_{S}(t)}[s \times(\hat{r} \times(x-\hat{b}))-\nabla w(\hat{\hat{b}}+\hat{r} \times(x-\hat{b}))] \sigma(w, q) n d \gamma \\
& +\dot{s} \cdot\left(\hat{J} \hat{r} \times s+g_{3}\right)+\ddot{c} g_{2}-\int_{\partial \widehat{\Omega}_{S}(t)}(\sigma(w, q) n) \cdot\left(g_{0, t}+(\hat{u} \cdot \nabla) g_{0}\right) d \gamma
\end{aligned}
$$

Using the continuity of the trace operator, we obtain

$$
\begin{gathered}
\int_{\widehat{\Omega}_{F}(t)}\left|w_{t}\right|^{2} d x+\frac{d}{d t} \int_{\widehat{\Omega}_{F}(t)}|\nabla w|^{2} d x+|\ddot{c}|^{2}+|\dot{s}|^{2} \\
\leq \varepsilon\left(\|w\|_{H^{2}\left(\widehat{\Omega}_{F}(t)\right)}^{2}+\|q\|_{H^{1}\left(\widehat{\Omega}_{F}(t)\right)}^{2}\right)+\widehat{C}_{\varepsilon}\left(\int_{\widehat{\Omega}_{F}(t)}|\nabla w|^{2} d x+\int_{\partial \widehat{\Omega}_{S}(t)}\left|g_{0, t}\right|^{2} d \gamma\right. \\
\left.\quad+\left\|g_{0}\right\|_{H^{2}\left(\widehat{\Omega}_{F}(t)\right)}^{2}+\int_{\widehat{\Omega}_{F}(t)}\left|g_{1}\right|^{2} d x+\left|g_{2}\right|^{2}+\left|g_{3}\right|^{2}+|s|^{2}\right)
\end{gathered}
$$

for $\varepsilon>0$ small enough. Now, we regard the equations for $w$ as a stationary system:

$$
\begin{cases}-\nabla \cdot \sigma(w, q)(t, x)=g_{1}(t, x)-w_{t}(t, x)-(\hat{u} \cdot \nabla) w(t, x), & x \in \widehat{\Omega}_{F}(t), \\ \nabla \cdot w(t, x)=0, & x \in \widehat{\Omega}_{F}(t), \\ w(t, x)=0, & x \in \partial \Omega, \\ w(t, x)=\dot{c}(t)+s(t) \times(x-\hat{b}(t))+g_{0}(t, x), & x \in \partial \widehat{\Omega}_{S}(t) .\end{cases}
$$

We can show that for a.e. $t \in(0, T)$, we have

$$
\begin{aligned}
\|w\|_{H^{2}\left(\widehat{\Omega}_{F}(t)\right)}+\|q\|_{H^{1}\left(\widehat{\Omega}_{F}(t)\right)} \leq & \widehat{C}\left(\left\|g_{0}\right\|_{H^{2}\left(\widehat{\Omega}_{F}(t)\right)}+\left\|g_{1}\right\|_{L^{2}\left(\widehat{\Omega}_{F}(t)\right)}\right. \\
& \left.+\left\|w_{t}\right\|_{L^{2}\left(\widehat{\Omega}_{F}(t)\right)}+\|\nabla w\|_{L^{2}\left(\widehat{\Omega}_{F}(t)\right)}+|\dot{c}|+|s|\right) .
\end{aligned}
$$

Indeed, let $\hat{\chi}_{e} \in C^{1}\left([0, T] ; C^{2}(\bar{\Omega})\right)$ be such that

$$
\left\{\begin{array}{l}
\hat{\chi}_{e}(t, y)=\hat{b}(t)+\hat{Q}(t) Q_{0}^{-1}\left(y-b_{0}\right) \quad \forall y \in \Omega_{S}(0), \\
\hat{\chi}_{e}(t, y)=y \quad \forall y \in \partial \Omega, \\
\exists \hat{\chi}_{e}^{-1} \in C^{1}\left([0, T] ; C^{2}(\bar{\Omega})\right): \hat{\chi}_{e}\left(t, \hat{\chi}_{e}^{-1}(t, x)\right)=x, \quad \forall t \in(0, T), \forall x \in \Omega, \\
\left\|\hat{\chi}_{e}-\mathrm{id}\right\|_{C^{1}\left([0, T] ; C^{2}(\bar{\Omega})\right)} \leq C\left(\|\hat{b}\|_{W^{1, \infty}(0, T)}+\|\hat{r}\|_{L^{\infty}(0, T)}\right) .
\end{array}\right.
$$

Then $\left(w \circ \hat{\chi}_{e}, q \circ \hat{\chi}_{e}\right)$ satisfies a stationary Stokes system in $\Omega_{F}(0)$. Here, we can apply classical estimates for the Stokes operator (see, for instance, [19]). For the right-hand side of the Stokes problem, we take into account that the terms of the form

$$
\left(\nabla \hat{\chi}_{e}-\mathrm{Id}\right)\left(D^{2} w \circ \hat{\chi}_{e}+\nabla q \circ \hat{\chi}_{e}\right)
$$


can be estimated in $L^{2}$ by $\varepsilon\left(\left\|w \circ \hat{\chi}_{e}\right\|_{H^{2}\left(\Omega_{F}(0)\right)}+\left\|q \circ \hat{\chi}_{e}\right\|_{H^{1}\left(\Omega_{F}(0)\right)}\right)$ in $\left(0, T_{0}\right) \times \Omega_{F}(0)$ provided that $T_{0}$ is chosen small enough in terms of $\|\hat{b}\|_{W^{1, \infty}(0, T)}+\|\hat{r}\|_{L^{\infty}(0, T)}$.

On the other hand, the divergence term equals $\left(\nabla w \circ \hat{\chi}_{e}\left(\nabla \hat{\chi}_{e}-\mathrm{Id}\right)\right)$, which is estimated in $H^{1}$ by $\varepsilon\left\|w \circ \hat{\chi}_{e}\right\|_{H^{2}\left(\Omega_{F}(0)\right)}$. Repeating this process $\left[T / T_{0}\right]+1$ times allows us to establish (69).

Finally, combining (69) with (67)-(68) and applying Gronwall's Lemma, we obtain the desired estimate (66).

Let us now establish the existence of more regular solutions when $g_{0} \equiv 0$. In order to do this, we suppose that $w_{0} \in H^{\varsigma}\left(\Omega_{F}(0)\right)$ for $\varsigma>5 / 2$ and we define some new functions. Set $J_{0}=J_{\mid t=0}$ and

$$
q_{1}:=-\left(\hat{u}_{\mid t=0} \cdot \nabla\right)\left(c_{1}+s_{0} \times\left(x-b_{0}\right)\right) 1_{\partial \Omega_{S}(0)}+\Delta w_{0}+g_{1 \mid t=0} \quad \text { on } \partial \Omega_{F}(0) .
$$

Then, we first define a triplet $\left(\tilde{c}_{1}, \tilde{s}_{0}, q_{0}\right)$ by

$$
\begin{aligned}
& \tilde{c}_{1}:=\frac{1}{m} \int_{\partial \Omega_{S}(0)} \sigma\left(w_{0}, q_{0}\right) n d \gamma+\frac{1}{m} g_{2}(0), \\
& \tilde{s}_{0}:=J_{0}^{-1}\left[\left(J_{0} r_{0}\right) \times s_{0}+\int_{\partial \Omega_{S}(0)}\left(x-b_{0}\right) \times \sigma\left(w_{0}, q_{0}\right) n d \gamma+g_{3}(0)\right]
\end{aligned}
$$

and

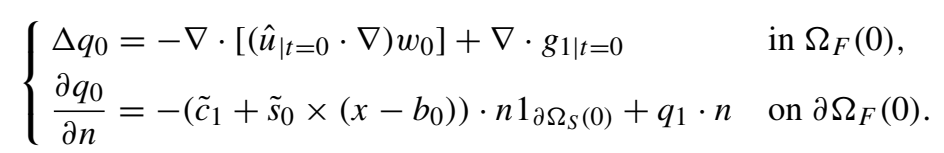

Using the fact that $J_{0}$ is positive definite, one can easily check that this system has a unique solution $\left(\tilde{c}_{1}, \tilde{s}_{0}, q_{0}\right)$ satisfying

$$
\begin{aligned}
& \left|\tilde{c}_{1}\right|+\left|\tilde{s}_{0}\right|+\left\|q_{0}\right\|_{H^{2}\left(\Omega_{F}(0)\right)} \\
& \quad \leq \widehat{C}\left(\left|s_{0}\right|+\left|c_{1}\right|+\left\|w_{0}\right\|_{H^{3}\left(\Omega_{F}(0)\right)}+\left\|g_{1}\right\|_{\hat{Y}_{0}}+\left\|g_{2}\right\|_{H^{1}(0, T)}+\left\|g_{3}\right\|_{H^{1}(0, T)}\right) .
\end{aligned}
$$

Finally,

$$
\tilde{w}_{0}:=g_{1 \mid t=0}+\nabla \cdot \sigma\left(w_{0}, q_{0}\right)-\left(\hat{u}_{\mid t=0} \cdot \nabla\right) w_{0} .
$$

Let us introduce the following compatibility condition:

$\tilde{w}_{0}(x)=\left(\tilde{c}_{1}+\tilde{s}_{0} \times\left(x-b_{0}\right)+\left[c_{2} \cdot \nabla\right]\left(c_{2}-w_{0}(x)\right)\right) 1_{\partial \Omega_{S}(0)}(x), \quad x \in \partial \Omega_{F}(0)$,

where we have denoted $c_{2}:=c_{1}+s_{0} \times\left(x-b_{0}\right)$.

Proposition 8. Let $g_{0} \equiv 0, g_{1} \in \hat{Y}_{0}$ and $g_{2}, g_{3} \in H^{1}(0, T)$. Assume that $w_{0} \in$ $H^{3}\left(\Omega_{F}(0)\right),\left(w_{0}, c_{0}, c_{1}, s_{0}, g_{1}, g_{2}, g_{3}\right)$ satisfy (8) and (71) and let $(\hat{u}, \hat{b}, \hat{r})$ satisfy (10) (with $\left(b_{0}, b_{1}, r_{0}\right)$ replaced by $\left.\left(c_{0}, c_{1}, s_{0}\right)\right)$ and $(11)-(12)$. Then, there exists $\widehat{C}$ (depending on $\Omega, \delta_{0}$ and $\left.\|\hat{u}\|_{\hat{Z}},\|\hat{b}\|_{H^{2}(0, T)},\|\hat{r}\|_{H^{1}(0, T)}\right)$ such that the solution of (65) satisfies

$$
(w, q, c, s) \in \hat{Y}_{2} \times\left(L^{2}\left(H^{3}\right) \cap H^{1}\left(H^{1}\right)\right) \times H^{3}(0, T) \times H^{2}(0, T)
$$


and

$\|(w, q, c, s)\|_{\hat{Y}_{2} \times\left(L^{2}\left(H^{3}\right) \cap H^{1}\left(H^{1}\right)\right) \times H^{3}(0, T) \times H^{2}(0, T)}$

$\leq \widehat{C}\left(\left\|g_{1}\right\|_{\hat{Y}_{0}}+\left\|g_{2}\right\|_{H^{1}(0, T)}+\left\|g_{3}\right\|_{H^{1}(0, T)}+\left\|w_{0}\right\|_{H^{3}\left(\Omega_{F}(0)\right)}+\left|c_{0}\right|+\left|c_{1}\right|+\left|s_{0}\right|\right)$.

Proof. Let us differentiate system (65) with respect to the time variable. This yields

$$
\begin{cases}w_{t t}(t, x)+(\hat{u} \cdot \nabla) w_{t}(t, x)-\nabla \cdot \sigma\left(w_{t}, q_{t}\right)(t, x)=\tilde{g}_{1}(t, x), & x \in \widehat{\Omega}_{F}(t), \\ \nabla \cdot w_{t}(t, x)=0, & x \in \widehat{\Omega}_{F}(t), \\ w_{t}(t, x)=0, & x \in \partial \Omega, \\ w_{t}(t, x)=\ddot{c}(t)+\dot{s}(t) \times(x-\hat{b}(t))+\tilde{g}_{0}(t, x), & x \in \partial \widehat{\Omega}_{S}(t), \\ m \dddot{c}(t)=\int_{\partial \widehat{\Omega}_{S}(t)}\left(\sigma\left(w_{t}, q_{t}\right) n\right)(t, x) d \gamma+\tilde{g}_{2}(t), & \\ (\hat{J} \ddot{s})(t)=((\hat{J} \hat{r}) \times \dot{s})(t)+\int_{\partial \widehat{\Omega}_{S}(t)}(x-\hat{b}(t)) \times\left(\sigma\left(w_{t}, q_{t}\right) n\right)(t, x) d \gamma+\tilde{g}_{3}(t), & \\ w_{t \mid t=0}=\tilde{w}_{0} \quad \text { in } \Omega_{F}(0), \quad \dot{c}(0)=c_{1}, \ddot{c}(0)=\tilde{c}_{1}, \dot{s}(0)=\tilde{s}_{0}, & \end{cases}
$$

where

$$
\begin{aligned}
& \tilde{g}_{1}:= g_{1, t}-\left(\hat{u}_{t} \cdot \nabla\right) w, \quad \tilde{g}_{0}:=(\hat{u} \cdot \nabla)(\dot{c}+s \times(x-\hat{b})-w), \\
& \tilde{g}_{2}:= g_{2, t}+\int_{\partial \widehat{\Omega}_{S}(t)}(\hat{u} \cdot \nabla) \sigma(w, q) n d \gamma+\int_{\partial \widehat{\Omega}_{S}(t)} \sigma(w, q)(\hat{r} \times n) d \gamma, \\
& \tilde{g}_{3}:=g_{3, t}-\dot{\hat{J}} \dot{s}-s \times \frac{d}{d t}(\hat{J} \hat{r})+\int_{\partial \widehat{\Omega}_{S}(t)}(\hat{r} \times(x-\hat{b})) \times \sigma(w, q) n d \gamma \\
& \quad+\int_{\partial \widehat{\Omega}_{S}(t)}(x-\hat{b}) \times(\hat{u} \cdot \nabla) \sigma(w, q) n d \gamma+\int_{\partial \widehat{\Omega}_{S}(t)}(x-\hat{b}) \times \sigma(w, q)(\hat{r} \times n) d \gamma .
\end{aligned}
$$

Observe now that, thanks to (12) and (71), we have

$$
w_{t \mid t=0}=\left(\ddot{c}(0)+\dot{s}(0) \times\left(x-b_{0}\right)+\tilde{g}_{0 \mid t=0}\right) 1_{\partial \Omega_{S}(0)} \quad \text { on } \partial \Omega_{F}(0) .
$$

This allows us to apply estimate (66) to (73):

$$
\begin{aligned}
&\left\|\left(w_{t}, q_{t}, \dot{c}, \dot{s}\right)\right\|_{\hat{Y}_{0} \times L^{2}\left(H^{1}\right) \times H^{2}(0, T) \times H^{1}(0, T)} \\
& \leq \widehat{C}\left(\left\|\tilde{g}_{0}\right\|_{L^{2}\left(H^{2}\right)}+\left\|\tilde{g}_{0}\right\|_{H^{1}\left(0, T ; L^{2}\left(\partial \widehat{\Omega}_{S}(t)\right)\right)}+\left\|\tilde{g}_{1}\right\|_{L^{2}\left(L^{2}\right)}+\left\|\tilde{g}_{2}\right\|_{L^{2}(0, T)}\right. \\
&\left.\quad+\left\|\tilde{g}_{3}\right\|_{L^{2}(0, T)}+\left\|\tilde{w}_{0}\right\|_{H^{1}\left(\Omega_{F}(0)\right)}+\left|c_{1}\right|+\left|\tilde{c}_{1}\right|+\left|\tilde{s}_{0}\right|\right) .
\end{aligned}
$$

Then, from classical estimates for the stationary Stokes system, we find

$\|(w, q, c, s)\|_{\hat{Y}_{2} \times\left(L^{2}\left(H^{3}\right) \cap H^{1}\left(H^{1}\right)\right) \times H^{3}(0, T) \times H^{2}(0, T)}$

$$
\begin{aligned}
\leq & \widehat{C}\left(\left\|\tilde{g}_{0}\right\|_{L^{2}\left(H^{2}\right)}+\left\|\tilde{g}_{0}\right\|_{H^{1}\left(0, T ; L^{2}\left(\partial \widehat{\Omega}_{S}(t)\right)\right)}+\left\|\tilde{g}_{1}\right\|_{L^{2}\left(L^{2}\right)}+\left\|\tilde{g}_{2}\right\|_{L^{2}(0, T)}\right. \\
& \left.+\left\|\tilde{g}_{3}\right\|_{L^{2}(0, T)}+\left\|\tilde{w}_{0}\right\|_{H^{1}\left(\Omega_{F}(0)\right)}+\left|c_{1}\right|+\left|\tilde{c}_{1}\right|+\left|\tilde{s}_{0}\right|\right) .
\end{aligned}
$$


Let us now estimate $\tilde{g}_{i}(0 \leq i \leq 3)$.

- Estimate of $\tilde{g}_{0}$. First,

$$
\left\|\tilde{g}_{0}\right\|_{L^{2}\left(H^{2}\right)} \leq C\|\hat{u}\|_{L^{2}\left(H^{2}\right)}\left(\|\dot{c}\|_{L^{\infty}(0, T)}+\|s\|_{L^{\infty}(0, T)}\right)+\|(\hat{u} \cdot \nabla) w\|_{L^{2}\left(H^{2}\right)} .
$$

For the last term in this inequality, we have, for $0<\delta<1 / 2$,

$$
\begin{aligned}
\|(\hat{u} \cdot \nabla) w\|_{L^{2}\left(H^{2}\right) \leq} \leq & C\left(\|\hat{u}\|_{L^{2}\left(H^{2}\right)}\|\nabla w\|_{C^{0}\left(C^{0}\right)}+\|\hat{u}\|_{C^{0}\left(H^{1}\right)}\|\nabla w\|_{L^{2}\left(W^{1, \infty}\right)}\right. \\
& \left.+\|\hat{u}\|_{C^{0}\left(H^{1}\right)}\|\nabla w\|_{L^{2}\left(W^{2,3}\right)}\right) \\
\leq & C\|\hat{u}\|_{\hat{Y}_{0}}\left(\|w\|_{C^{0}\left(H^{5 / 2+\delta}\right)}+\|w\|_{L^{2}\left(H^{7 / 2+\delta}\right)}+\|w\|_{L^{2}\left(H^{7 / 2}\right)}\right. \\
\leq & \varepsilon\left(\|w\|_{C^{0}\left(H^{3}\right)}+\|w\|_{L^{2}\left(H^{4}\right)}\right)+\widehat{C}_{\varepsilon}\left(\|w\|_{C^{0}\left(H^{2}\right)}+\|w\|_{L^{2}\left(H^{2}\right)}\right)
\end{aligned}
$$

for any $\varepsilon>0$. Then, we use

$$
\tilde{g}_{0, t}(t, x)=[(\hat{u} \cdot \nabla)(\dot{c}+s \times(x-\hat{b})-w)]_{t}(t, x), \quad x \in \widehat{\Omega}_{F}(t) .
$$

Taking traces in this identity and using (12), for $0<\delta<1 / 2$ we deduce

$\left\|\tilde{g}_{0}\right\|_{H^{1}\left(0, T ; L^{2}\left(\partial \widehat{\Omega}_{S}(t)\right)\right)}$

$$
\begin{aligned}
\leq & C\|\hat{u}\|_{H^{1}\left(0, T ; L^{2}\left(\partial \widehat{\Omega}_{S}(t)\right)\right)}\left(\|\dot{c}\|_{L^{\infty}(0, T)}+\|s\|_{L^{\infty}(0, T)}+\|w\|_{C^{0}\left([0, T] ; L^{\infty}\left(\partial \widehat{\Omega}_{S}(t)\right)\right)}\right) \\
& +C\|\hat{u}\|_{C^{0}\left([0, T] ; L^{\infty}\left(\partial \widehat{\Omega}_{S}(t)\right)\right)}\left(\|\dot{c}\|_{H^{1}(0, T)}+\|s\|_{H^{1}(0, T)}+\|w\|_{H^{1}\left(H^{3 / 2+\delta}\right)}\right) \\
\leq & \widehat{C}_{\varepsilon}\left(\|c\|_{H^{2}(0, T)}+\|s\|_{H^{1}(0, T)}+\|w\|_{L^{\infty}\left(H^{1}\right)}+\|w\|_{H^{1}\left(L^{2}\right)}\right) \\
& +\varepsilon\left(\|w\|_{C^{0}\left(H^{3}\right)}+\|w\|_{H^{1}\left(H^{2}\right)}\right)
\end{aligned}
$$

for any $\varepsilon>0$.

- Estimate of $\tilde{g}_{1}$. For $0<\delta<1 / 2$ we have

$$
\begin{aligned}
\left\|\left(\hat{u}_{t} \cdot \nabla\right) w\right\|_{L^{2}\left(L^{2}\right)} & \leq\left\|\hat{u}_{t}\right\|_{L^{2}\left(L^{2}\right)}\|\nabla w\|_{C^{0}\left(C^{0}\right)} \leq \widehat{C}\|w\|_{C^{0}\left(H^{5 / 2+\delta}\right)} \\
& \leq \widehat{C}_{\varepsilon}\|w\|_{C^{0}\left(H^{1}\right)}+\varepsilon\|w\|_{C^{0}\left(H^{3}\right)}
\end{aligned}
$$

for any $\varepsilon>0$.

- Estimate of $\tilde{g}_{2}$. Using (12), we obtain

$$
\begin{aligned}
\left\|\tilde{g}_{2}\right\|_{L^{2}(0, T)} \leq & \left(\|\hat{u}\|_{C^{0}\left([0, T] ; L^{\infty}\left(\partial \widehat{\Omega}_{S}(t)\right)\right)}+\|\hat{r}\|_{L^{\infty}}\right)\left(\|w\|_{L^{2}\left(H^{5 / 2+\delta}\right)}\right. \\
& \left.+\|q\|_{L^{2}\left(H^{3 / 2+\delta}\right)}\right)+\left\|g_{2}\right\|_{H^{1}(0, T)}
\end{aligned}
$$

for any $0<\delta<1 / 2$. Thus,

$$
\begin{aligned}
\left\|\tilde{g}_{2}\right\|_{L^{2}(0, T)} \leq & \widehat{C}_{\varepsilon}\left(\|w\|_{L^{2}\left(H^{2}\right)}+\|q\|_{L^{2}\left(H^{1}\right)}+\left\|g_{2}\right\|_{H^{1}(0, T)}\right) \\
& +\varepsilon\left(\|w\|_{L^{2}\left(H^{4}\right)}+\|q\|_{L^{2}\left(H^{3}\right)}\right)
\end{aligned}
$$

for any $\varepsilon>0$. 
- Estimate of $\tilde{g}_{3}$. Analogously as for $\tilde{g}_{2}$, we easily obtain

$$
\begin{aligned}
\left\|\tilde{g}_{3}\right\|_{L^{2}(0, T)} \leq \widehat{C}_{\varepsilon}\left(\|w\|_{L^{2}\left(H^{2}\right)}+\|q\|_{L^{2}\left(H^{1}\right)}+\left\|g_{3}\right\|_{H^{1}(0, T)}+\|s\|_{H^{1}(0, T)}\right) \\
+\varepsilon\left(\|w\|_{L^{2}\left(H^{4}\right)}+\|q\|_{L^{2}\left(H^{3}\right)}\right)
\end{aligned}
$$

for any $\varepsilon>0$.

Reassembling estimates (76)-(81), and combining them with (75), we find

$$
\begin{aligned}
& \|(w, q, c, s)\|_{\hat{Y}_{2} \times\left(L^{2}\left(H^{3}\right) \cap H^{1}\left(H^{1}\right)\right) \times H^{3}(0, T) \times H^{2}(0, T)} \\
& \leq \widehat{C}_{\varepsilon}\left(\|w\|_{\hat{Y}_{0}}+\|q\|_{L^{2}\left(H^{1}\right)}+\|c\|_{H^{2}(0, T)}+\|s\|_{H^{1}(0, T)}+\left\|g_{1}\right\|_{\hat{Y}_{0}}+\left\|g_{2}\right\|_{H^{1}(0, T)}\right. \\
& \left.\quad+\left\|g_{3}\right\|_{H^{1}(0, T)}+\left\|w_{0}\right\|_{H^{3}\left(\Omega_{F}(0)\right)}+\left|c_{1}\right|+\left|\tilde{c}_{1}\right|+\left|\tilde{s}_{0}\right|\right)+\varepsilon\|(w, q)\|_{\hat{Y}_{2} \times\left(L^{2}\left(H^{3}\right) \cap H^{1}\left(H^{1}\right)\right)}
\end{aligned}
$$

for any $\varepsilon>0$. Applying Proposition 7 in order to estimate the first four terms and taking $\varepsilon$ small enough, we obtain the desired inequality (72).

Corollary 9. Let $k_{0} \in[0,2] \backslash\{1 / 2\}$. Let $g_{0} \equiv 0, g_{1} \in L^{2}\left(H^{2-k_{0}}\right) \cap H^{1-k_{0} / 2}\left(L^{2}\right)$ and $g_{2}, g_{3} \in H^{1-k_{0} / 2}(0, T)$. Assume that $w_{0} \in H^{3-k_{0}}\left(\Omega_{F}(0)\right),\left(w_{0}, c_{0}, c_{1}, s_{0}, g_{1}, g_{2}, g_{3}\right)$ satisfy (8) and condition (71) if $k_{0}<1 / 2$. Furthermore, let $(\hat{u}, \hat{b}, \hat{r})$ satisfy (10) (with $\left(b_{0}, b_{1}, r_{0}\right)$ replaced by $\left.\left(c_{0}, c_{1}, s_{0}\right)\right)$ and (11)-(12). Then there exists $\widehat{C}$ (depending on $\Omega, \delta_{0}$ and $\left.\|\hat{u}\|_{\hat{Z}},\|\hat{b}\|_{H^{2}(0, T)},\|\hat{r}\|_{H^{1}(0, T)}\right)$ such that the solution of (65) satisfies

$$
(w, q, c, s) \in \hat{Y}_{2-k_{0}} \times\left(L^{2}\left(H^{3-k_{0}}\right) \cap H^{1-k_{0} / 2}\left(H^{1}\right)\right) \times H^{3-k_{0} / 2}(0, T) \times H^{2-k_{0} / 2}(0, T)
$$

and

$$
\begin{aligned}
& \|(w, q, c, s)\|_{\hat{Y}_{2-k_{0}} \times\left(L^{2}\left(H^{3-k_{0}}\right) \cap H^{1-k_{0} / 2}\left(H^{1}\right)\right) \times H^{3-k_{0} / 2}(0, T) \times H^{2-k_{0} / 2}(0, T)} \\
& \leq \widehat{C}\left(\left\|g_{1}\right\|_{\hat{Y}_{-k_{0}}}+\left\|g_{2}\right\|_{H^{1-k_{0} / 2}(0, T)}+\left\|g_{3}\right\|_{H^{1-k_{0} / 2}(0, T)}+\left\|w_{0}\right\|_{H^{3-k_{0}\left(\Omega_{F}(0)\right)}}+\left|c_{0}\right|+\left|c_{1}\right|+\left|s_{0}\right|\right) .
\end{aligned}
$$

The proof of this corollary is classical and it relies on interpolation arguments between Proposition 7 (with parameter $k_{0} / 2$ ) and Proposition 8 (with parameter $1-k_{0} / 2$ ) (we refer to [18] and [1]).

\section{References}

[1] Bergh, J., Löfström, J.: Interpolation Spaces. An Introduction. Grundlehren Math. Wiss. 223, Springer, Berlin (1976) Zbl 0344.46071 MR 0482275

[2] Borchers, W., Sohr, H.: On the equations $\operatorname{rot} \mathbf{v}=\mathbf{g}$ and div $\mathbf{u}=\mathbf{f}$ with zero boundary conditions. Hokkaido Math. J. 19, 67-87 (1990) Zbl 0719.35014 MR 1039466

[3] Boulakia, M., Guerrero, S.: A regularity result for a solid-fluid system associated to the compressible Navier-Stokes equations. Ann. Inst. H. Poincaré Anal. Non Linéaire 26, 777-813 (2009) Zbl 1177.35146 MR 2526402

[4] Boulakia, M., Osses, A.: Local null controllability of a two-dimensional fluid-structure interaction problem. ESAIM Control Optim. Calc. Var. 14, 1-42 (2008) Zbl 1149.35068 MR 2375750 
[5] Conca, C., San Martín, J., Tucsnak, M.: Existence of solutions for the equations modelling the motion of a rigid body in a viscous fluid. Comm. Partial Differential Equations 25, 1019-1042 (2000) Zbl 0954.35135 MR 1759801

[6] Desjardins, B., Esteban, M. J.: On weak solutions for fluid-rigid structure interaction: compressible and incompressible models. Comm. Partial Differential Equations 25, 1399-1413 (2000) Zbl 0953.35118 MR 1765138

[7] Doubova, A., Fernández-Cara, E.: Some control results for simplified one-dimensional models of fluid-solid interaction. Math. Models Methods Appl. Sci. 15, 783-824 (2005) Zbl 1122.93008 MR 2139944

[8] Fabre, C., Lebeau, G.: Prolongement unique des solutions de l'équation de Stokes. Comm. Partial Differential Equations 21, 573-596 (1996) Zbl 0849.35098 MR 1387461

[9] Fernández-Cara, E., Guerrero, S., Imanuvilov, O. Yu., Puel, J.-P.: Local exact controllability of the Navier-Stokes system. J. Math. Pures Appl. 83, 1501-1542 (2004) Zbl pre02164954 MR 2103189

[10] Fursikov, A. V., Imanuvilov, O. Yu.: Controllability of Evolution Equations. Lecture Notes Ser. 34, Seoul National Univ. (1996) Zbl 0862.49004 MR 1406566

[11] Giga, Y., Sohr, H.: Abstract $L^{p}$ estimates for the Cauchy problem with applications to the Navier-Stokes equations in exterior domains. J. Funct. Anal. 102, $72-94$ (1991) Zbl 0739.35067 MR 1138838

[12] Grandmont, C., Maday, Y.: Existence for an unsteady fluid-structure interaction problem. Math. Model. Numer. Anal. 34, 609-636 (2000) Zbl 0969.76017 MR 1763528

[13] Imanuvilov, O. Yu.: Remarks on exact controllability for the Navier-Stokes equations. ESAIM Control Optim. Calc. Var. 6, 39-72 (2001) Zbl 0961.35104 MR 1804497

[14] Imanuvilov, O. Yu., Takahashi, T.: Exact controllability of a fluid-rigid body system. J. Math. Pures Appl. (9) 87, 408-437 (2007) Zbl 1124.35056 MR 2317341

[15] Nakoulima, O.: Contrôlabilité à zéro avec contraintes sur le contrôle. C. R. Math. Acad. Sci. Paris 339, 405-410 (2004) Zbl 1060.93015 MR 2092753

[16] San Martín, J., Starovoitov, V., Tucsnak, M.: Global weak solutions for the two-dimensional motion of several rigid bodies in an incompressible viscous fluid. Arch. Ration. Mech. Anal. 161, 113-147 (2002) Zbl 1018.76012 MR 1870954

[17] Takahashi, T.: Analysis of strong solutions for the equations modeling the motion of a rigid-fluid system in a bounded domain. Adv. Differential Equations 8, 1499-1532 (2003) Zbl 1101.35356 MR 2029294

[18] Tartar, L.: An Introduction to Sobolev Spaces and Interpolation Spaces. Springer (2007) Zbl 1126.46001 MR 2328004

[19] Temam, R., Navier-Stokes Equations. Theory and Numerical Analysis. Rev. ed., Stud. Math. Appl. 2, North-Holland, Amsterdam (1979) Zbl 0426.35003

[20] Zeidler, E.: Nonlinear Functional Analysis and Its Applications. I. Fixed-Point Theorems. Springer, New York (1986) Zbl 0583.47050 MR 0816732 